\title{
SCRIVERE E LEGGERE NEL CHIOSTRO: LO SCRIBA-MONACHUS
}

\section{SIMONA GAVINELLI}

\author{
UDC: $27-788-055.1$ \\ 27-25"05/11" \\ Original scientific paper \\ Manuscript received: 15. 11. 2016. \\ Revised manuscript accepted: 15. 02. 2017. \\ DOI: 10.1484/J.HAM.5.113733
}

S. Gavinelli

Dipartimento di Studi Medievali, Umanistici e Rinascimentali Università Cattolica del Sacro Cuore Largo Agostino Gemelli, 1 20123 Milano, Italia simona.gavinelli@unicatt.it

The paper particularly analyzes the monastic culture in Early Middle Ages. A specific focus is made on the evidence of monastic status, with use of 'monachus' in subscriptions of mediaeval manuscripts before the $12^{\text {th }}$ century.

Keywords: Middle Age, monastic culture, monastic scribes, monastic subscriptions

Come doverosa premessa preciso che, data la vastità del tema, per quanto cronologicamente circoscritto entro il secolo XII, in questo contributo mi limiterò a proporre schegge esemplificatrici (per lo più sufficientemente note) in modo da caratterizzare le linee portanti di un percorso culturale molto sfaccettato nel tempo e nello spazio.

Nella vita di s. Anselmo di Aosta (1033-1109), stesa dall'allievo Eadmero e poi sintetizzata da Giovanni di Salisbury (1120-1180), sono narrate le ultime fasi compositive del Proslogion, concluso tra il 1077 e il 1078 mentre era priore dell'abbazia normanna di Notre-Dame du $\mathrm{Bec}^{1}$. Appaiono particolarmente suggestivi i passaggi biografici che rievocano il frangente redazionale dell'argomento ontologico (secondo la definizione di Immanuel Kant), finalizzato alla dimostrazione aprioristica dell'esistenza di Dio, quando la serrata speculazione teologica gli aveva tolto il sonno e l'appetito, convogliando inoltre su di lui le scontate invidie della comunità, puntualmente trasformate in manifestazioni demoniache. L'apice era stato raggiunto con la sparizione delle tavolette dove, durante una veglia notturna, aveva appuntato alcune intuizioni che, anche dopo una seconda ricopiatura, erano state ritrovate sul pavimento con la cera raschiata, ma il circuito malefico si era tuttavia interrotto solo grazie a un terzo tentativo (nel richiamo numerologico alla perfezione trinitaria), coincidente con la trasposizione del testo sulla pergamena (in chartis).

Alcuni scatti di vita monastica quotidiana, che fissano «in imagine» le sequenze tecniche della preparazione libraria, vengono invece offerti dal frontespizio dell'Ambrogio, $D e$ officiis ministrorum Bamberg, Staatsbibliothek, Ms. Patr. 5 (B-II-5), f. 1v, copiato alla metà del secolo XII presso il chiostro benedettino di Michelsberg della medesima città ${ }^{2}$. Nel riquadro centrale della miniatura troneggia l'arcangelo Michele, osservato dal chiostro da alcuni monaci, mentre un altro pictor dipinge la sua effigie sul raccordo architettonico di un timpano, come particolare evocativo del monastero; la cornice, nei moduli figurativi dei clipei classici di tradizione imperiale, accoglie una sequenza di dieci giovani monaci, didascalicamente intenti a illustrare le fasi della preparazione del libro e del suo impiego scolastico, squadernando dunque le fatiche artigiane della lisciatura, della rigatura e della scrittura sulla pergamena, fino all'adattamento della legatura, includendo l'uso delle tavolette cerate per le annotazioni cursorie?.

Pare comunque che la litigiosità fosse quasi un topos del monachesimo occidentale, come un'inclinazione deteriore della fragile natura umana, già denunciata verso la fine del sec. IV da Gerolamo in molte epistole e soprattutto nel

\footnotetext{
'Abbreviazioni: CLA = E.A. LOWE, Codices Latini Antiquiores, I-XII e Suppl., Oxford, 1934-1971; Colophons = BÉNÉDICTINS DU BOUVERET, Colophons de manuscrits occidentaux des origines au XVIe siècle, I-VI, Fribourg (Suisse), 1965; J. J. JOHN = J.J. JOHN, The named (and namable) scribes in Codices Latini Antiquiores, in E. Condello - G. De Gregorio (a cura di), Scribi e colofoni: le sottoscrizioni di copisti dalle origini all'avvento della stampa. Atti del Seminario di Erice (23-28 ottobre 1993) =X Colloquio del Comité international de paléographie latine, Spoleto, 1995 (Biblioteca del Centro per il collegamento degli studi medievali e umanistici in Umbria, 14), p. 107-121; Manuscrits datés, VII = C. SAMARAN - R. MARICHAL, Catalogue des manuscrits en écriture latine portant des indications de date, de lieu ou de copiste, VII: Ouest de la France et pays de Loire, Paris, 1984.

GIOVANNI Di SALISBURY, Anselmo e Becket. Due vite, a cura di I. Biff, Milano, 1990, p. 49-50. Il monaco Eadmero chiarisce invece che Anselmo, rappresentante del filone teologico platonico-agostiniano, al Bec avrebbe scritto solo il Monologion (1076) e il Proslogion, posticipando il resto della sua produzione alla permanenza arcivescovile di Canterbury: EADMERO DI CANTERBURY, Vita di Sant'Anselmo, a cura di S. Gavinelli, Milano, 1987, p. 17, 57-59. La contestualizzazione sul periodo di 'pacificazione spirituale' al Bec è presentata da: I. BIFFI, Anselmo d’Aosta e dintorni: Lanfranco, Guitmondo, Urbano II, Milano, 2007, p. 1-34.

${ }^{2}$ R.M.W. STAMMBERGER, Scriptor und scriptorium. Das Buch im Spiegel mittelalterlicher Handschriften, Graz, 2003 (Lebensbilder des Mittelalter), S. 40-41. ${ }^{3}$ K. SCHREINER, Vergine, madre, regina: i volti di Maria nell'universo cristiano, Traduzione di C. Miglio, Roma, 1995, p. 100-103.
} 
Tractatus de psalmo 119 dove, tra l'ironico e l'angosciato, contestava ai suoi monaci - che pure avevano avuto il coraggio di abbandonare la patria, la famiglia e il patrimonio -, la tendenza ad alterarsi per frivolezze, come per il possesso di una penna ( $«$... et propter calamum rixam facimus in monasterio»); ma l'esemplificazione conferma indirettamente una diffusa familiarità con le attività grafiche e intellettuali ${ }^{4}$. Agli inizi del secolo V Giovanni Cassiano (36o-435), nel passaggio di Institutiones, IV 12, lascia del resto intendere che i monaci potessero ricopiare i manoscritti «intra cubilia sua», cioè portandoli nel dormitorio accanto ai giacigli, specificando tuttavia che, al segnale convenuto per la preghiera, tutti dovessero prontamente interrompere ogni attività, e chi fosse preposto all'opus scriptoris era tenuto quindi a sospendere l'esecuzione di una lettera o di un apice. Dal tardo-antico i pueri oblati e i giovani monaci erano dunque abituati a impiegare libri e strumenti scrittori, in linea con la Regola di s. Benedetto che, sotto la rubrica «Si quid debeant monachi proprium habere» (e poi nel cap. XXXIII sui limiti della proprietà individuale), vietava perfino il possesso di un libro, di una tavoletta o di uno stilo («nullam omnino rem, neque codicem neque tabulas neque graphium»), se non dietro la concessione temporanea dell'abate e per uso comunitario5. Ancora più esplicito è il cap. XLVIII della stessa Regola, che stabiliva la proporzione quotidiana del lavoro manuale e della preghiera (l'opus Dei cadenzato nelle ore del giorno) mentre in Quaresima i monaci, dal mattino all'ora terza, leggevano sotto sorveglianza alcuni testi presi dalla bibliotheca (talvolta interpretata come la Bibbia) e che venivano distribuiti all'inizio del tempo penitenziale o alla domenica ${ }^{6}$. Nell'anonima Regula Magistri (più prolissa della Regola di s. Benedetto e ascrivibile a un autore vissuto forse in Gallia nel secolo VII), si fa riferimento a un'arca «cum diversis codicibus, membranis et cartis monasterii», cioè a una biblioteca-archivio di efficacia funzionale dove la denominazione di 'arca' richiama la terminologia vete- ro-testamentaria, più spesso connessa con la conservazione delle reliquie santorali. Ritroviamo il termine a Bobbio, una delle prime fondazioni longobarde riconducibili al monachesimo itinerante dell'irlandese s. Colombano, sorta verso il 614 con l'appoggio del re Agilulfo (590-616) e della cattolica Teodolinda (589-628) come saldo avamposto contro i domini bizantini, pur nelle contrapposizioni religiose tra cattolicesimo e arianesimo acuite dallo scisma tricapitolino ${ }^{7}$. A Bobbio i successori di Colombano, provenienti probabilmente dall'abbazia borgognona di Luxeuil (590), erano anche animati da un intenso fervore riorganizzativo sul piano librario visto che Attala (615-627) viene segnalato per la sua sollecitudine nel rilegare i libri del cenobio: «libros ligaminibus firmare $»^{8}$. La medesima bibliofilia fu condivisa dai successori Boboleno (639-652) e Vorgusto (secolo VII seconda metà), che potevano in effetti disporre di arcae per i libri procurati ${ }^{9}$. La conferma deriva dalle annotazioni in corsiva poste su tre monumenti patristici scritti in onciale e semionciale del secolo VII, stellarmente dispersi tra la Biblioteca Ambrosiana di Milano, la Biblioteca Apostolica Vaticana e l'Archivio di Stato di Torino, in cui si specifica «(Liber) de arca domno (sic) Atalani (Bobuleni, Vorgusti)», là dove Bobbio era pure diventato un deposito di materiale codicologico suscettibile di riscrittura palinsesta ${ }^{10}$. Assecondando le aspettative papali il monastero aveva in effetti incrementato il proprio arsenale bibliografico con opere dogmatiche antiereticali, come gli atti dei concili di Efeso (431) e di Calcedonia (451), ma aveva promosso anche lo studio dei testi grammaticali per l'apprendimento del latino, soprattutto grazie alla componente irlandese $\mathrm{e}^{\mathrm{I}}$.

Larca bobbiese sembra del resto richiamata in forma iconografica nella miniatura del profeta Esdra della Bibbia Amiatina Firenze, Biblioteca Medicea Laurenziana, Amiatino 1 (Codex Amiatinus), f. 5r, copiata in onciale inglese agli inizi del secolo VIII' ${ }^{12}$. Il collegamento, anche ideologico, era con i modelli aulici continentali del monastero italo-meri-

\footnotetext{
${ }^{4}$ S. HIERONYMI PRESBYTERI Tractatus de psalmo CXVIIII, in SANCTI HIERONYMI PRESBITERI Tractatus sive homiliae in Psalmos, in Marci evangelium aliaque argumenta, ed. G. Morin, Maredsoli, 1897 (Anecdota Maredsolana, 3/2), p. 231.

${ }_{5}^{5}$ S. PRICOCO, La regola di san Benedetto, in Id. (a cura di), La regola di san Benedetto e le Regole dei Padri, Milano, 1995 (Scrittori greci e latini), p. 200201; su questi giovani monaci, vd. G. ARCHETTI, «Sub virga magistri». Custodia e disciplina nell'educazione carolingia dei pueri oblati, in Studi medievali, s. III, 57/2 (2016), p. 527-582.

${ }^{6}$ «omnes singulos codices de bibliotheca quos per ordinem ex integro legant»: S. PRICOCO, op. cit. (n. 5), p. 224-227.

${ }^{7}$ M. PACAUT, Monaci e religiosi nel Medioevo, Bologna, 1989, p. 64-69. Per i vari problemi: M. TOSI, Arianesimo Tricapitolino norditaliano e Penitenza privata Iroscozzese: due piste importanti per riprendere la questione critica delle opere di Colombano, in Archivum Bobiense, 10-11, 1988-1989, p. 9-118.

${ }^{8}$ N. SCIVOLETTO, Filologia e cultura latina, Roma, 2000, p. 416.

${ }_{9}$ G. CAVALLO, Libri e cultura nelle due Italie longobarde, in C. Bertelli - G.P. Brogiolo (a cura di), Il futuro dei Longobardi. L'Italia e la costruzione dell'Europa di Carlo Magno. Saggi, Milano, 2000, p. 85-103: 86.

${ }^{10} \mathrm{Ci}$ si riferisce al Milano, Biblioteca Ambrosiana, $\mathrm{S} 45$ sup., palinsesto di una Bibbia gotica (CLA III 365); al Città del Vaticano, Biblioteca Apostolica Vaticana, lat. 5758 con i Sermones di s. Agostino in onciale (CLA I 36); quindi alla miscellanea patristica Torino, Archivio di Stato, IB.II.27 (CLA IV 438), contenente l'Epitome delle Divinae Institutiones di Lattanzio, Origene, Ilario di Poitiers: B. BISCHOFF, Paleografia latina. Antichità e medioevo. Ed. italiana a cura di G. P. Mantovani - S. Zamponi, Padova, 1992 (Medioevo e umanesimo, 81), p. 273; N. SCIVOLETTO, op. cit. (n. 8), p. 416; L. SCAPPATICCI, Codici e liturgia a Bobbio: testi, musica e scrittura. Secoli X ex.-XII, Città del Vaticano, 2008, p. 19.

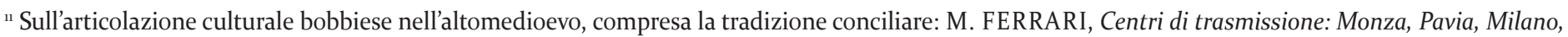
Bobbio, in G. BILLANOVICH-M. FERRARI, La trasmissione dei testi nell'Italia nord-occidentale, in La cultura antica dell'Occidente latino dal VII all'XI secolo, Spoleto, 1975 (Settimana di studio del Centro Italiano di Studi sull'Altomedioevo, 22), p. 303-320: 313-320; K. ZECHIEL-ECKES, Die Concordia canonum des Cresconius. Studien und Edition, Frankfurt am Main, 1992 (Freiburger Beiträge zur mittelalterlichen Geschichte, 5), S. 87-91; S. GAVINELLI, Testi agiografici e collezioni canoniche in età carolingia attraverso codici dell'Ambrosiana, in M. Ferrari - M. Navoni (a cura di), Nuove ricerche su codici in scrittura latina dell'Ambrosiana. Atti del Convegno, Milano, 6-7 ottobre 2005, Milano, 2007 (Bibliotheca erudita. Studi e documenti di storia e filologia, 31), p. 53-78: 74-75.

${ }^{12}$ CLA III 299; D.H. WRIGHT, Some notes on English Uncial, in Traditio, 17, 1961, p. 441-456: 452-454; A. PETRUCCI, L'Onciale Romana. Origini, sviluppo e diffusione di una stilizzazione grafica altomedievale (secc. VI-IX), in Studi medievali, s. III, 12/1, 1971, p. 75-132: 120-127; J.J. G. ALEXANDER, Insular Manuscripts from the 6 th to the 9 th Century, London, 1978 (Survey of Manuscripts Illuminated in the British Isles, 1), p. 32-35; B. FISCHER, Lateinische Bibelhandschriften im frühen Mittelalter, Freiburg im Br., 1985, S. 9-34, 67-69, 130-132, 180-181, 354-363; J. GRIBOMONT, Cassiodore et ses Bibles latines, in
} 
dionale di Vivarium, creato verso il 540-545 da un Cassiodoro amareggiato dagli eventi posteriori alla successione del re ostrogoto Teodorico († 526), e pertanto deciso a configurare un'ambiziosa progettualità culturale di ispirazione classica, declinata però in chiave monastica sul De doctrina christiana di Agostino ${ }^{13}$. La Bibbia Amiatina appare menzionata perfino nell'Historia Abbatum, II, 15 di Beda il Venerabile (672-735) quando allude alla propria biblioteca monastica che l'abate Ceolfrido († 716) aveva arricchito con «tres pandectes novae translationis» (di cui due disperse), richieste ai monasteri gemelli di Wearmouth e Jarrow, una delle quali da recapitare in dono al papa in occasione del suo pellegrinaggio ad limina Apostolorum, durante il quale trovò la morte ${ }^{14}$. In questa Bibbia grandior, modellata dunque sulle Pandette cassiodoriane, il profeta Esdra - alle cui spalle si staglia appunto un'arca-armadio simile a un'edicola con i libri biblici sui ripiani -, è proposto nell'atto dello scrivere ${ }^{15}$. Innestandosi dunque nella tradizione classica si intendeva pertanto esaltare non solo l'auctor, ma anche il ruolo manuale dello scriptor (o del miniatore) attraverso un recupero figurativo antropomorfo e monumentale che verrà poi assunto come postulato normativo nella miniatura di lusso degli evangeliari anglosassonici e carolingi ${ }^{16}$.

In un quadro storico, dominato dalle risacche della crisi economica e dal dilagante analfabetismo, un ulteriore espediente iconografico enfatizza un circoscritto episodio di produzione libraria monastica della fine del secolo VII, che fu soprattutto orchestrato da una committenza verticistica come l'autorità papale. Nell'antico cimelio della Regula pastoralis di Gregorio Magno (590-604) Ivrea, Biblioteca
Capitolare, I (1), di piccolo formato ma elegantissimo nell'uso della minuscola di Luxeuil, e con decorazioni architettoniche di matrice tardo-antica, una duplice scacchiera sistemata tra colonne a tinte tenui (nello stile degli esametri incatenati) propone la formula propiziatoria «DESIDERIUS PAPA / VIVAT DIU» (f. iv), che consente di identificare il presule Desiderio di Ivrea tra i firmatari del sinodo romano del 680 indetto da papa Agatone (678-681) in previsione del VI Concilio ecumenico costantinopolitano, destinato a comporre l'eresia monotelita ${ }^{17}$. Da qui l'ipotesi di David Ganz che, in maniera più che condivisibile, evidenzia come la stilizzazione della minuscola di Luxeuil possa avere avuto un forte impulso quantitativo proprio per iniziativa pontificia quando papa Agatone sarebbe ricorso al prestigio grafico della medesima abbazia per commissionare un blocco di libri-simbolo in grado di suggellare la ricompattazione tra il Papato e i vescovi, soprattutto quelli dell'Italia settentrionale, geograficamente più esposti ai rischi 'scismatici' tricapitolini ${ }^{18}$.

Un fenomeno analogo, per quanto con maggiore partecipazione istituzionale, si verificò poi durante la riforma ecclesiastica iniziata nel secolo XI-XII da Gregorio VII (1073-1085): per coordinamento papale anche nei complessi canonicali più periferici, insieme al rilancio della vita comune, furono inoltrati programmaticamente dei libri come manifesto del nuovo corso, a partire dalle voluminose Bibbie atlantiche (nella definizione di Pietro Toesca) che, secondo una produzione 'modulare', erano commissionate presso le fondazioni monastiche toscane o umbro-laziali (soprattutto vallombrosane) con la conseguente stilizzazione grafica e

\footnotetext{
Flavio Magno Aurelio Cassiodoro. Atti della Settimana di Studi, Cosenza-Squillace 1983, Cosenza-Catanzaro, 1986, p. 262-280; V. LONGO - S. MAGRINI - M. PALMA, Bibliografia della Bibbia Amiatina (1990-1999), Roma, 2000.

${ }^{13}$ In rapporto all'ampia bibliografia sulla produzione epistolare (Variae) ed esegetica di Cassiodoro mi limito a: A. MOMIGLIANO, Cassiodoro, in Dizionario Biografico degli Italiani, XXI, Roma, 1978, p. 494-504; S. BJORNIE, The Letter Collection of Cassiodorus, in C. Sogno - B. K. Storin - E. J. Watts (edd.), Late antique Collections. A critical introduction and reference guide, Oakland, 2007, p. 433-448; L. CRACCO RUGGINI, Cassiodorus and the Practical Sciences, in Vivarium in Context, Vicenza, 2008, p. 23-53; F. CARDINI, Cassiodoro il Grande. Roma, i barbari e il monachesimo, Milano, 2009. Per l'attività più propriamente grafica e filologica: F. TRONCARELLI, Un codice emendato da Cassiodoro?, in Scrittura e Civiltà, 9, 1985, p. 147-168; ID., Litteras pulcherrimas. Correzioni di Cassiodoro nei codici di Vivarium, in Scrittura e civiltà, 20, 1996, p. 89-109; ID., Vivarium: i Libri, il Destino, Turnhout, 1998 (Instrumenta Patristica, 33); CASSIODORO, De orthographia. Tradizione manoscritta, fortuna, edizione critica a cura di P. Stoppacci, Firenze, 2010, p. XXXVII-XLV.

${ }^{14}$ VENERABILIS BEDAE Historia Ecclesiastica gentis Anglorum, Historia Abbatum et Epistola ad Ecgbertum cum epistola Bonifacii ad Cudberthum, ed. C.G.H. Moberly, Oxonii, 1881, p. 384.

${ }^{15}$ B. BISCHOFF, Paleografia latina, op. cit. (n. 10), p. 264. Un'attenta interpretazione dell'immagine di Esdra induceva Pierre Courcelle a riconoscere nell'armarium alle spalle del profeta i nove volumi della grande Bibbia «novae translationis», suddivisa per «cola et commata», indicata da Cassiodoro in Institutiones, I 13, per cui ne supponeva la disponibilità presso lo scriptorium di Jarrow al momento della redazione, mentre con una brillante ipotesi Bonifatius Fischer coglieva nello stesso ritratto la rappresentazione di tutte e tre le tipologie bibliche eseguite a Vivarium: nell'armarium la Bibbia in nove volumi, in primo piano, confuse tra gli strumenti scrittori sparsi sul pavimento, le Pandette di piccolo formato scritte «minutiore manu» (Institutiones, I 12), e finalmente il Codex grandior, tenuto dal profeta sulle ginocchia: P. COURCELLE, Les Lettres grecques en Occident. De Macrobe à Cassiodore, Paris, $1948^{2}$ (Bibliothèque des Écoles françaises d'Athènes et de Rome, 159 bis), p. 356-362; B. FISCHER, Codex Amiatinus und Cassiodor, in Biblische Zeitschrift, N.F., 6, 1962, S. 57-79.

${ }^{16}$ O. PÄCHT, La miniatura medievale. Una introduzione, Torino, 1987, p. 173-183; F. CRIVELLO, Eadfrith: l'arte libraria nelle isole britanniche, in E. Castelnuovo (a cura di), Artifex bonus - Il mondo dell'artista medievale, Roma-Bari, 2004, p. 12-19.

${ }^{17}$ CLA III 300; M. FERRARI, Libri e testi prima del Mille, in G. Cracco (a cura di), Storia della Chiesa di Ivrea dalle origini al XV secolo, Roma, 1998 (Chiese d'Italia, 1), p. 511-533: 511-517; D. GANZ, Texts and scripts in surviving manuscripts in the script of Luxeuil, in P. Ní Chatháin - M. Richter (edd.), Ireland and Europe in the Early Middle Age. Texts and transmission, Dublin, 2001, p. 186-204.

${ }^{18}$ D. GANZ, The Luxeuil Prophets and Merovingian missionary Strategies, in R.G. Babcock (ed.), Beinecke Studies in Early Manuscripts, in Yale University Library Gazette, Suppl. to vol. 66, 1991, p. 105-117; D. GANZ, Texts and scripts, op. cit. (n. 17), p. 190, 200. Allo stesso nucleo apparterrebbe infatti anche l'analoga copia gregoriana, di formato tendenzialmente quadrato, dei Moralia in Iob (libri XXVII-XXXXV) Verona, Biblioteca Capitolare, XL (38), nella parte non palinsesta, dove compare lo stesso rubricatore del codice eporediese: M. FERRARI, Libri e testi, op. cit. (n. 17), p. 516; cfr. CLA IV 497-501; A. SPAGNOLO, I manoscritti della Biblioteca Capitolare di Verona, a cura di S. Marchi, Verona, 1996, p. 92-93; L.G.G. Ricci (a cura di), Gregorio Magno e l'invenzione del Medioevo, Firenze, 2006 (Archivum Gregorianum, 9), p. 74-75 n. 1 (scheda di F. Crivello). Nel 1968 dal codice veronese sono stati staccati i fascicoli palinsesti dalla cui scriptio inferior (ff. 205-206, 211-216, 219-224, 227-231, 234-241, 243-266) sono emersi preziosi frammenti in capitale rustica e in onciale del sec. V con stralci di Virgilio, Livio ed Euclide, ma la differente origine paleografica, estesa all'Italia, ha suggerito un possibile interscambio con Bobbio di materiale pergamenaceo di riuso: B. BISCHOFF, Manuscripts and Libraries in the age of Charlemagne, Cambridge, 1994 (Cambridge Studies
} in Palaeography and Codicology, 1), p. 16. 
decorativa ${ }^{19}$. Per trascinamento pure i passionari, gli omeliari e il Decretum di Burcardo di Worms (950-1025) rientravano nella stessa configurazione codicologica delle Bibbie atlantiche e, una volta esportati in Italia settentrionale come pegni sensibili di questa politica ecclesiale centralizzata, influivano spesso sullo stile degli scriptoria di approdo ${ }^{20}$. Nel clima di contrapposizione tra Papato e Impero la Chiesa aveva dunque investito in modo consistente sulla valenza simbolica del libro di fattura monastica proprio per sottolineare un'esibita appartenenza di campo ${ }^{21}$.

Per isolare i copisti di chiara formazione monastica ho quindi condotto una prima ricognizione attraverso i repertori più accreditati, in primis quello dei Colophons raccolti dai Bénédictins du Bouveret, e ho provato ad assumere come indice dellautocoscienza di ruolo le qualifiche di monachus o di religiosus esplicitate con evidenza nelle sottoscrizioni dei manoscritti, in genere di carattere liturgico o patristico-esegetico ${ }^{22}$. Anche l'indagine sui Codices Latini Antiquiores ha comunque confermato come le sottoscrizioni altomedievali rimangano rare, lasciando dunque prevalere l'ottica ascetica della «fuga saeculi» e dell'annullamento della propria individualità, che induceva pertanto a giustificare la dura fatica dello scrivere come un sacrificio motivato escato- logicamente "pro remedio animae»" 23 : su novantadue copisti anteriori al secolo IX solo in quarantotto casi viene dichiarato lo stato sociale (clericale o monastico), che diventa invece più consistente nelle formulazioni basso-medievali quando anche le donne, quasi sempre di ambito religioso, salgono percentualmente al $14 \%$. Nella mia rassegna finale, collocata in appendice, ho quindi preferito omettere le attestazioni (in realtà la maggioranza) in cui il contesto monastico è arguibile, o scontato, sulla base della provenienza dei codici o della loro committenza.

Tra i meriti di Gregorio Magno, papa-monaco e missionario per eccellenza, si aggiunge quello di essere stato un sollecito promotore del proprio magistero attraverso la diffusione manoscritta delle sue opere, veicolando dunque le risonanze grafiche dell'onciale romana ${ }^{24}$. Pur con scarso chiaroscuro tale stile scrittorio viene in effetti riproposto come richiamo evocativo (suggerito forse dall'antigrafo) nei Dialogi di Gregorio Magno Milano, Biblioteca Ambrosiana, B 159 sup. dove, oltre a numerose iniziali a motivi zoomorfi, si individua la preziosa sottoscrizione dell'unico copista Giorgione, priva però di precisazioni di status monastico: «Expliciunt Dialogorum Gregorii papae urbis Romae libri numero IIII. Deo favente Anastasio abbate iovente (sic), Georgione peccatore scrivente....»5. Come valido ancoraggio

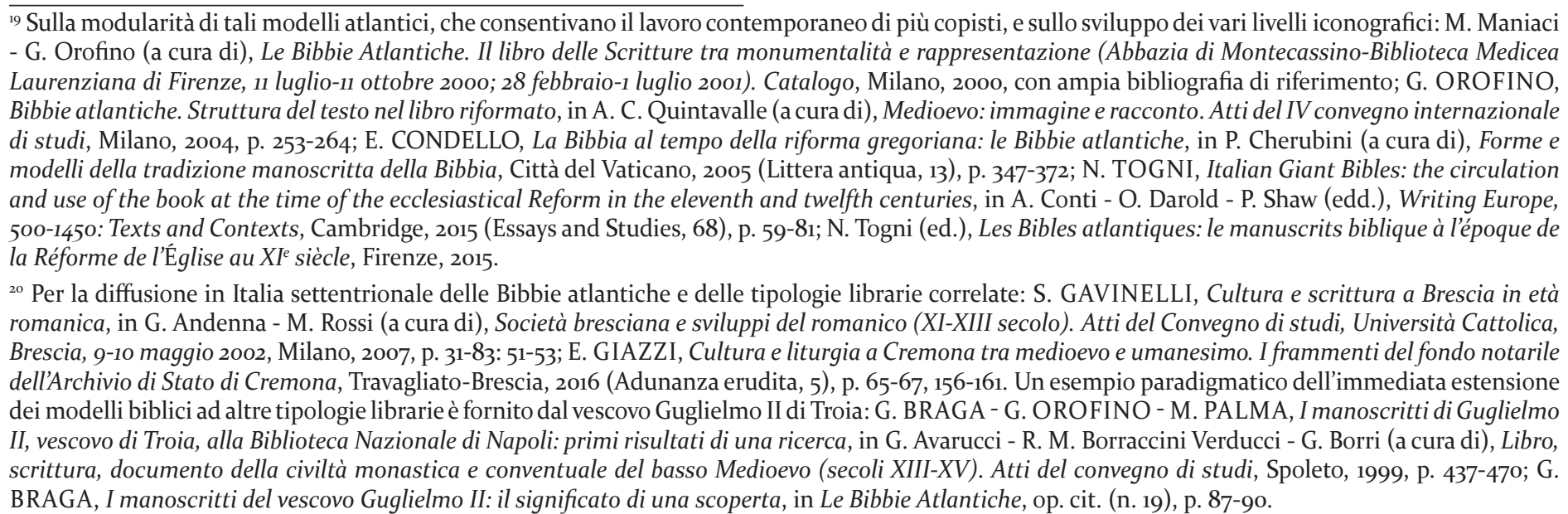

${ }^{21}$ O. CAPITANI, La riforma gregoriana, in Le Bibbie Atlantiche, op. cit. (n. 19), p. 7-13.

${ }^{22}$ Ho condotto l'esame limitandomi a: Colophons; J. J. JOHN. Ai fini della ricerca non è stata utile la consultazione di L. REYNHOUT, Formules latines de colophons, I-II, Turnhout, 2006 (Bibliologia. Elementa ad librorum studia pertinentia, 25, A-B), p. 85-100 che, nel capitolo Le temps des monastères, prende solo in considerazione le formule finali più antiche come «Sicut navigantibus portus...» $\mathrm{o}$ «Scribere qui nescit...». In generale sugli scribi altomedievali: A. PETRUCCI, Alfabetismo ed educazione grafica degli scribi altomedievali (secc. VII-IX), in D. Ganz (ed.), The Role of the book in medieval Culture, I, Turnhout, 1986, p. 109-131.

${ }^{23}$ J. J. JOHN, p. 113; P. SUPINO MARTINI, Il libro e il tempo, in E. Condello - G. De Gregorio (a cura di), Scribi e colofoni: le sottoscrizioni di copisti dalle origini all'avvento della stampa. Atti del Seminario di Erice (23-28 ottobre 1993) = X Colloquio del Comité international de paléographie latine, Spoleto, 1995 (Biblioteca del Centro per il collegamento degli studi medievali e umanistici in Umbria, 14), p. 235-266: 235-245. La scarsità di sottoscrizioni altomedievali emerge anche dalla catalogazione in progress dei Manoscritti datati d'Italia: T. DE ROBERTIS, La catalogazione dei manoscritti datati d'Italia, in M. Marchiaro - S. Zamponi (a cura di), Conoscere il manoscritto: esperienze, progetti, problemi. Dieci anni del Progetto Codex in Toscana. Atti del Convegno internazionale, Firenze, 29-30 giugno 2006, Firenze, 2007, p. 125-143: 128, 133-135. Sull'educazione scrittoria femminile, più avanzata nelle aree germaniche anche prima del secolo XII: A. I. BEACH, Women as Scribes: book production and monastic reform in twelfth-century Bavaria, Cambridge, 2004, p. 9-23; N. GIOVẼ, Donne che non lasciano traccia. Presenze e mani femminili nel documento altomedievale, in Agire da donna. Modelli e pratiche di rappresentazione (secoli VI-X). Atti del convegno (Padova, 18-19 febbraio 2005), Turnhout, 2007, p. 189-209.

${ }_{24}$ Per la precoce diffusione delle opere di Gregorio Magno: A. Petrucci (a cura di), Codex Trecensis: la "Regola pastorale" di Gregorio Magno in un codice del VI-VII secolo: Troyes, Médiathèque de l’Agglomération Troyenne, 504, I-II, Firenze, 2005 (Archivium Gregorianum, 5); P. CHIESA, Oltre il Codex Trecensis. Nuove prospettive a partire dalla 'Regula pastoralis', in C. Leonardi (a cura di), Gregorio Magno e le origini dell'Europa, Firenze, 2014, p. $291-306$. Sulla diffusione della scrittura onciale, in particolare del tipo romano: A. PETRUCCI, L'Onciale Romana. Origini, sviluppo e diffusione di una stilizzazione grafica altomedievale (secc. VI-IX), in Studi medievali, s. III, 12/1, 1971/1, p. 75-134; N. GIOVÈ, Caratteri e trasformazioni nella cultura scritta nel V secolo, P. Delogu - S. Gasparri (a cura di), Le trasformazioni del V secolo. L'Italia, i barbari e l'Occidente romano. Atti del seminario di Poggibonsi, 18-20 ottobre 2007, Turnhout, 2010, p. 679-703: 698-699.

${ }_{25}^{25}$ Sul codice, particolarmente autorevole per la moderna constitutio del testo critico: CLA III 309; J. J. JOHN, p. 117; P. COLLURA, Studi paleografici. La precarolina e la carolina a Bobbio, Milano, 1943, p. 33, 48, 74-75, 165, 188; G. CAVALLO, op. cit. (n. 9), p. 86; L. CASTALDI, Per un'edizione critica dei «Dialoghi» di Gregorio Magno: ricognizioni preliminari, in Filologia mediolatina, 10, 2003, p. 1-39; L. G. G. RICCI, op. cit. (n. 18), p. 79-81 n. 3 (scheda di L. Castaldi). 
cronologico nel colophon viene poi inclusa la committenza dell'abate Anastasio, il cui nome compare in un diploma del 747 emanato dal re longobardo Ratchis (744-749 e 756-757).

Emblematico per la modalità della trascrizione, ripartita tra più scribi, è il Livio, $A b$ Urbe condita, XXII-XXX Città del Vaticano, Biblioteca Apostolica Vaticana, Reg. lat. 762, un raro storiografo romano esemplato a Tours nel secolo VIII-IX in uno dei monasteri di punta per la tipizzazione morfologica dell'innovativa scrittura carolina, dove però il gruppo dei monaci-copisti «Aldo e Ansoaldus e Fredegaudus, Gyslarus, Landemarus, Nautus» si sottoscrive sempre senza alcuna qualifica ${ }^{26}$. Tra i primi classici a essere eseguiti durante l'abbaziato di Alcuino di York (796-804) documenta al tempo stesso un caso paradigmatico del rapporto tra antigrafo tardo-antico e apografo carolingio, visto che fu copiato dal Paris, Bibliothèque Nationale de France, lat. 7530 (Puteanus), un autorevole monumento in onciale degli inizi del secolo $\mathrm{V}^{27}$.

Anche i giovani Tattone e Grimaldo (quest'ultimo proiettato a ricoprire la carica di potente abate di S. Gallo tra l'841 e l'872) sembra che trovino superfluo rideterminarsi nel ruolo monastico quando si nominano nell'epistola indirizzata nell'817 al precettore Regimberto con cui, nel St. Gallen, Stiftsbibliothek, 914, p. 202-203, accompagnavano l'archetipo della Regola di s. Benedetto - (il caposaldo della riforma monastica carolingia appena varata nel concilio di Aquisgrana dell'817) -, che era stato ricopiato a Reichenau in minuscola alamannica direttamente dal modello cassinese inoltrato da Paolo Diacono $(† 799)^{28}$.

Nelle sottoscrizioni dei manoscritti la qualifica sacerdotale prevale talvolta sullo status monastico, come suggerisce il celebre copista Winitharius di S. Gallo - probabilmente preposto alla direzione dello scriptorium monastico nel momento del particolarismo grafico della minuscola alamanna prima dell'adeguamento carolino (conseguito verso il primo terzo del secolo IX) -, il quale, nel suo Liber collectaneus St. Gallen, Stiftsbibliothek, 238, contenente i Dicta Winithari (in prevalenza estratti biblici e isidoriani) preferisce sottoscriversi denominandosi solo come presbiter ${ }^{29}$. Nel codice sangallese il suo nome è infatti inserito due volte, prima in vivaci linee alternate rosse e verdi «Explicit problema Uuinitharii presbiteri qui hunc librum scripsit et complevit a fine usque in finem + omnis qui simul legerit oret pro eo qui scripsit si Dominum habeat propiciatorem. Amen, alleluia» (p. 181), poi in semplice minuscola bruna «Deo et Christo gloria quia explicet liber quem Uuinitharius peccator et inmerito ordinatus presbiter scripsit ex suo proprio labore Deo auxiliante perfecit et non est hic nec unus folius quem ille de suo labore non adquississet aut conparando aut mendicando. Et non est in hoc libro unus apex aut iota una quem manus eius non pinxisset» (p. 493) $3^{30}$.

Analogo è il caso dell'abate Maiolo di Cluny (954-994) il quale, nel colophon del Rabano Mauro Super Hieremiam London, British Library, Additional 22820, realizzato presso l'abbazia borgognona, appare formalizzato come committente del manufatto attraverso la iussio diretta al copista Herimannus, che preferisce tuttavia fregiarsi della sola funzione sacerdotale: «iussu domni Maiolis abbatis ab Herimanno sacerdote ... Sancto Petro Cluniensi coenobio» ${ }^{31}$.

In tale prospettiva un'altra illustre esemplificazione documentaria è fornita dal monastero del Mont Saint-Michel in Normandia, ricostruito nel 961 dopo l'invasione normanna,

${ }^{26}$ CLA I 109; E. K. RAND, A Survey of the Manuscript of Tours, I-II, Cambridge (Mass.), 1929 (The Mediaeval Academy of America Publications, 3 ), I, p. 97; II, pl. XXVI, 3; B. BISCHOFF, Manuscripts and Libraries, op. cit. (n. 18), p. 75, 133 (con un elenco di diciotto manoscritti classici scritti a Tours nel sec. IX, di cui molti a carattere grammaticale).

${ }^{27}$ CLA IX 560; J. J. JOHN, p. 111, 116-118; P. BUSONERO, Alcune osservazioni sul rapporto tra antigrafo e apografo nella tradizione della terza decade di Livio, in Nuovi annali della Scuola speciale per archivisti e bibliotecari, 18, 2004, p. 19-36. Per la ripartizione del lavoro di copia: B. VICTOR, Simultaneous copying of classical texts 800-1100. Techniques and their consequences, in H. Spilling (cur.), La collaboration dans la production de l'écrit médiéval. Actes du XIII ${ }^{e}$ colloque du Comité international de paléographie latine (Weingarten, 22-25 septembre 200o), Paris, 2003 (Matériaux pour l'histoire publiés par l'Ecole des Chartes, 4), p. 347-358; V.I. MAZHUGA, La tradition carolingienne tourangelle des Histories de Grégoire de Tours vue à travers les fragments de Saint Pétersbourg, Copenhague et Wolfenbüttel, in Scriptorium, 62/1, 2008, p. 113-121: 119 pl. 17b; M. B. PARKES, Their Hands Before Our Eyes. A Closer Look at Scribes, Aldershot, 2008 (Lyell Lectures, 1999), p. 8.

${ }^{28}$ L. GILISSEN, Observations codicologiques sur le codex Sangallensis 914, in P. Cockshaw - M.-C. Garand - P. Jodogne (edd.), Miscellanea codicologica F. Masai dicata MCMLXXXIX, I, Gent, 1979 (Publications de Scriptorium, 8), p. 51- 70; BENEDICTUS DE NURSIA Regula Benedicti: de codice 914 in Bibliotheca Monasterii S. Galli servato (ff. $1 r-86 v$ [85v] = p. 1-172; saec. IX), quam simillime expressa, addita descriptione et paginis et versibus congruente (G. MORIN - A. AMELLI) necnon praefatione palaeographica B. BISCHOFF auctore, St. Ottilien, 1983, p. XII-XIV; S. PRICOCO, op. cit. (n. 5), p. XLIVXLVIII; R. BERGMANN - S. STRICKER, Katalog der althochdeutschen und altsächsischen Glossenhandschriften, I, Berlin, 2005, S. 595; S. GAVINELLI, Modelli librari e formazione ideologica centralizzata, in Carlo Magno e le Alpi. Atti del XVIII Congresso internazionale di studio sull'alto medioevo, Susa, 19-20 ottobre 2006-Novalesa, 21 ottobre 2006, Spoleto, 2007, p. 105-139: 124. I monasteri alamanni di Reichenau e di S. Gallo erano stati fondati sulle principali vie di transito della regione alpina anche con funzione di presidio difensivo: G. ANDENNA, Monasteri e canoniche regolari delle Alpi, in E. Castelnuovo - F. De Gramatica (a cura di), Il Gotico nelle Alpi: 1350-1450. Catalogo,Trento, 2002, p. 79-89: 79-81 in cui a p. 80 menziona il manoscritto sangallese della Regola benedettina.

${ }^{29}$ B. BISCHOFF, Manuscripts and Libraries, op. cit. (n. 18), p. 37.

${ }^{30}$ CLA VII 934; J. J. JOHN, p. 121; A. VON EUW, Die St. Galler Buchkunst vom 8. bis zum Ende des 11. Jahrhunderts, I: Textband, St. Gallen, 2008 (Monasterium Sancti Galli, 3), S. 301-303 n. 6. Grazie alla sottoscrizione la sua grafia viene riconosciuta, per intero o in parte, in alcuni manoscritti sangallesi della seconda metà del secolo VIII, i St. Gallen, Stiftsbibliothek, 2 (una Bibbia con estratti dal Vecchio e dal Nuovo Testamento), 11 (excerpta biblici e Gregorio Magno, Homiliae in Ezechielem), 70 (Epistulae paoline), 109 (Gerolamo, In Psalmos), 225 (miscellanea di opere isidoriane e Eucherio di Lione), 907 (miscellaneo, contenente glosse etimologiche e brani cronografici e biblici), infine il 1399a2 (Antifonario): CLA VII 893a, 894, 896, 906, 928, 952, 996; J. J. JOHN, p. 121; J. C. MARTIN IGLESIAS, Réflexions sur la tradition manuscrite de trois oeuvres d'Isidore de Séville: le De natura rerum, la Regula monachorum et le De origine Getarum, Vandalorum, Sueborum, in Filologia mediolatina, 11, 2004, p. 205-263; A. VON EUW, Die St. Galler Buchkunst, op. cit. (Ibidem), p. 297-301 n. 1-5; V. VON BÜREN, La transmission du De Moribus du ps Sénèque, de Winithar de S. Gall à Sedulius Scottus, in P. Farmhouse Alberto - D. Paniagua (édd.), Ways of Approaching Knowledge in Late Antiquity and the Early Middle Ages: Schools and Scholarship, Nordhausen, 2012 (Studia classica et mediaevalia, 8), p. 206-244; B. BISCHOFF, Katalog der festländischen Handschriften des neunten Jahrhunderts: III: Padua-Zwickau, Wiesbaden, 2014, S. 298-299, 303, 306, 315 n. 5502a, 5505a, 5544a, 5568a 5666a, $5871 a$.

${ }^{31}$ A. G. WATSON, Catalogue of Dated and Datable Manuscripts c. 700-16oo in the Department of Manuscripts the British Library, I, London, 1979, n. 278, pl. 18; P. SAENGER, Space between words. The origins of silent reading, Stanford, 1997, p. 215; E.H. AUBERT, Nova cluniacensia: les feuillets de garde du manuscrit Paris BnF nal 1236 et la création de la liturgie clunisienne, in Bucema. Bulletin du centre d'études médiévales. Auxerre, 12, 2012, par. 37. Dallo 


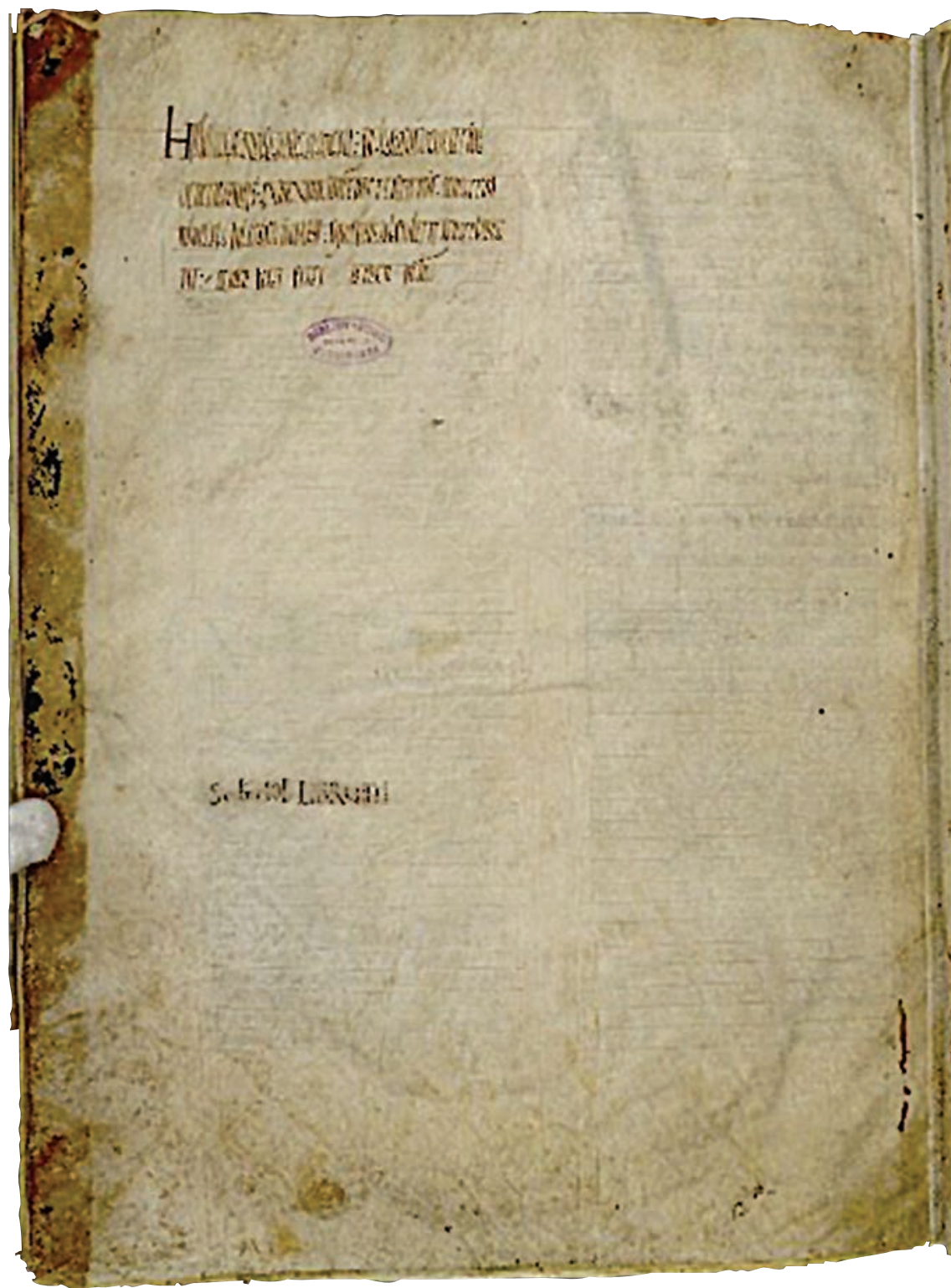

Tav. I. Gregorio Magno, Moralia in Iob (libri XVIII-XXXV) Avranches, Bibliothèque Municipale, $98, f .227 v$, sec. $X-X I$. e il cui patrimonio librario verso la metà del secolo XIX venne trasferito alla Bibliothèque Municipale del vicino centro di Avranches ${ }^{32}$. Della produzione manoscritta compresa tra il secolo X-XII sopravvivono trentasei codici, realizzati da una quindicina di scribi che esibiscono i vari stadi evolutivi della scrittura carolina e un ventaglio decorativo di capilettere a ramages fortemente segnati dall'influsso anglosassonico 33 . Ancora durante l'abbaziato di Mainardo II (circa 991-1009) due diversi copisti si divisero la compilazione grafica di un paio di voluminosi tomi dei Moralia in Iob di Gregorio Magno, che impaginarono in un'elegante mise en page bicolonnare ${ }^{34}$. I primi libri I-XVII sono trasmessi dal codice Avranches, Bibliothèque Municipale, 97 che, pur rovinato nei fogli finali, risulta scritto dalla stessa mano del coevo Avranches, Bibliothèque Municipale, 111, con un testo esegetico di Aimone di Auxerre, e di un ulteriore manoscritto storiografico, ora diviso in due, cioè il Chronicon di Adone di Vienne del Paris, Bibliothèque Nationale de France, 5920 e il De gestis Francorum di Gregorio di Tours (unito ancora al Chronicon di Adone di Vienne) della copia Leiden, Bibliotheek der Rijksuniversiteit, Voss. lat. F 39, ff. 1r-8ov e 81r-135v, l'unica che esibisca un chiaro colophon sul f. 135v: qui il monaco Hervardus, restituito dalle assonanze del verso leonino, è tuttavia menzionato solo come frater: «Scripsit unus librarius qui se nominat frater Hervardus» ${ }^{35}$. La seconda porzione dei Moralia in Iob (libri XVIII-XXXV) è invece conservata nell'Avranches, Bibliothèque Municipale, 98 dove, sul finale f. $227 \mathrm{v}$, insieme all'anatema per chi vorrà sottrarre il volume, una sottoscrizione in alfabeto misto dai tratti cancellereschi e con litterae elongatae, riporta il nome di Gualterius che iniziò l'opera di trascrizione poi proseguita per la massima parte

studio di M.-C. GARAND, Copistes de Cluny au temps de saint Maieul (948-994), in Bibliothèque de l'école des chartes, 136, 1978, p. 5-36, su ventisette manoscritti copiati da otto notai monastici del sec. X solo tre presentano il colophon.

${ }^{32}$ P. SAENGER, op. cit. (n. 31), p. 208-209.

${ }^{33}$ Sono quasi tutti depositati alla Bibliothèque Municipale di Avranches, tranne due fondamentali testi liturgici come il Sacramentario New York, Pierpont Morgan Library, M.641, scritto a piena pagina verso il 1060 con tracce della notazione musicale più antica e connotato da dodici ampie miniature a foglio intero per le maggiori festività, e la Bibbia romanica Bordeaux, Bibliothèque Municipale (Médiathèque), 1, eseguita verso il 1070 mentre una parte (ff. 45 ) è conservata a Rouen, Bibliothèque Municipale, MS 116: C. SAMARAN - R. MARICHAL, Catalogue des manuscrits en écriture latine portant des indications de date, de lieu ou de copiste, VI: Bourgogne, Centre, Sud-Est et Sud-Ouest de la France, Paris, 1968, p. 510; J.J.G. ALEXANDER, Norman illumination at Mont St. Michel, 966-110o, Oxford, 1970, p. 77-82, 183- 209, 212-213, 226-228, 240-242, 243-245, pl. 12d, 13, 31-36, 38, 40-46, 50a-c, 51a, e, 52b, c, e, 53b, e, f, 54a-b, e, 55a-e, 131, 135; ID., A Romanesque copy from Mont-Saint-Michel of an initial in the Corbie Psalter, e F. AVRIL, La décoration des manuscrits du Mont-Saint-Michel, XI-XII siècles, in Millénaire monastique du Mont-Saint-Michel, I-II, Paris, 1967, rispettivamente I, p. 243 e II, p. 213 et 226-229 ill. 121123; W. CAHN, La Bible romane. Chefs-d'œuvre de l'enluminure, Fribourg-Paris, 1982, p. 115, 268 cat. 55, fig. 72, p. 115; M. DOSDAT, L'enluminure romane au Mont Saint-Michel, X'-XII siècles, Avranches-Rennes, 1991, p. 58, 60, 61; fig. 7, 8; B.C. RAW, Anglo-Saxon crucifixion iconography and the art of the monastic revival, Cambridge-New York, 1991, p. 230. Sui manoscritti micaelici si veda anche da ultimo: J. L. LESERVOISIER, Les manuscrits du Mont Saint-Michel, Avranches, 2006.

34 P. SAENGER, op. cit. (n. 31), p. 208.

35 K.A. DE MEYER, Codices Vossiani Latini, I: Codices in Folio, Leiden, 1973, p. 84-86; Manuscrits datés, VII, p. 67, 71 pl. X-XI; F. Boccini (a cura di), Bibliotheca Gregorii Magni manuscripta. Censimento dei manoscritti di Gregorio Magno e della sua fortuna (epitomi, florilegia, agiografie, liturgia). Census of manuscripts of Gregory the Great and his fortune (Epitomes, Anthologies, Hagiographies, Liturgy), 1: (Aachen-Chur), Firenze, 2015 (Biblioteche e Archivi, 29. Bibliotheca Gregorii Magni manuscripta, 1), p. 48 n. 318. Deve invece essere rettificata la presenza come scriba di Gosselenus monachus indicata in Colophons, II 5547 per il codice carolingio Avranches, Bibliothèque Municipale, 108, f. 126v, in cui diverse mani della Francia centrale o settentrionale della seconda metà del sec. IX hanno assemblato il Liber scintillarum e il Commentarius in epistulas Pauli in quanto la semplice formula «Gotlenus monachus» costituisce un'aggiunta forse del sec. X: B. BISCHOFF, Katalog der festländischen Handschriften des neunten Jahrhunderts, I: Aachen-Lambach, Wiesbaden, 1998, S. 42 n. 180 
dal monaco Martinus, entrambe figure identificabili in una lista monastica del Mont Saint-Michel corrispondente all'abbaziato di Mainardo II ${ }^{36}$ : «Hic est liber Sancti Michaelis archangeli, inchoatus a Gualterio, venerabili viro, ex maxima parte scriptus a Martino monacho in loco Sancti Iuliani. Si quis eum abstulerit anathema sit. Amen. Fiat. Fiat. Amen in Domino» [Tav. I].

Un ulteriore scriba del monastero micaelico è Gyraldus, attivo tra la metà e il terzo quarto del secolo XI e che, senza qualifica monastica, si sottoscrive in due codici di opere agostiniane, le Enarrationes in Psalmos, e il Contra Faustum, rispettivamente Avranches, Bibliothèque Municipale, 77, f. $238 \mathrm{v}$ e 90, f. $185 \mathrm{v}$ (qui in versi leonini rossi e verdi) ${ }^{37}$. In un'articolata sottoscrizione complessiva il suo nome compare di nuovo nell'Avranches, Bibliothèque Municipale, 91, f. $175 \mathrm{~V}$ insieme alla semplice menzione di altri due monaci, Varinus e Rannulfus (che si ripete in lettere capitali al $\mathrm{f}$. 234v), tutti e tre preposti alla stesura piuttosto omogenea degli Opuscola di Agostino anteriori di qualche decennio ${ }^{38}$. Il tipo di sottoscrizione è accostabile ai versi leonini in capitale maiuscola a linee alternate rosse e verdi che concludono il codice Avranches, Bibliothèque Municipale, 103: sul f. $220 \mathrm{~V}$ sono riportati i nomi di sei copisti (Gualterius, Hilduinus, Scollandus, Ermenaldus, Osbernus, e Nicholaus) che, prima del 1072, con impeccabile uniformità, hanno copiato le Homeliae XL in evangelia sempre di Gregorio Magno (hom. 1-25, $24,26,27,29,28,30-40$ ), nessuno dei quali si caratterizza però come monachus ${ }^{39}$. Tra costoro Osberno che si firma appunto brevemente, senza qualificarsi nei versi leonini dell'Avranches, Bibliothèque Municipale, 163, f. 77r con le Vitae patrum seu historia Lausiaca e s. Agostino Liber de magistro ad Adeodatum ${ }^{40}$.

Nell'Avranches, Bibliothèque Municipale, 72, f. Iv le stesse movenze grafiche in inchiostro verde e rosso contraddistinguono il contenuto della ricca miscellanea patristica in cui sono riunite alcune opere di Gerolamo (Adversus Iovinianum come esortazione alla verginità e l'Altercatio Luciferiani et orthodoxi), Agostino (Contra Felicianum arianum) e Ambrogio (De bono mortis), e che fu eseguita tra il 1040 e il 1055 con poche iniziali ornate; il nome del copista, il monaco Frotmundus, di probabile origine britannica, è inserito in un breve testo poetico sul f. $199 \mathrm{v}$ dove, senza precisazione di ruolo, esordisce con la formula: «Hunc studuit totum / Frotmundus scribere librum...» ${ }^{41}$.

Nel corso del lungo abbaziato di Robert de Torigni (11541186), dopo un cinquantennio di crisi politico-religiosa, il monastero micaelico recuperò una certa stabilità economica che garantì il rinnovamento architettonico e la ripresa dell'attività scrittoria, documentata da almeno una dozzina di codici indigeni, oltre al magnifico cartulario monastico Avranches, Bibliothèque Municipale, 210 ${ }^{42}$.

Diventa comunque superfluo ribadire il grande contributo apportato dalla componente monastica alla riforma carolingia fin dal primo magistero di Alcuino e dei suoi collaboratori che, anche attraverso una fitta corrispondenza epistolare, costruì una rete di personalità di calibro in grado di condividere un progetto filologico di studi biblico-teologici ed esegetici corroborato dalla creatività poetica ${ }^{43}$. Monaco era sicuramente l'irlandese Dungal, trasferito dall'abbazia regia di St. Denis a Pavia per riorganizzare le scuole ecclesiastiche del regno italico, come indica il noto capitolare di Corteolona dell'825, e di cui si conservano, per quanto non sottoscritti, i suoi interventi autografi nell'allora rarissimo testo di Lucrezio (Oblongus) Leiden, Bibliotheek der Rijksuniversiteit, Voss. lat. F. 30, impiegato per argomentare alcune questioni astronomiche sulle eclissi solari dell'810 su richiesta dello stesso Carlo Magno ${ }^{44}$.

I tratti dell'autografia nel contesto dell'assemblamento librario sono rilevabili anche nella ricca miscellanea del St. Gallen, Stiftsbibliothek, 878, il vademecum personale che Walafrido Strabone († 849) si era fatto redigere a Reichenau tra l'825 e l'849 grazie all'ausilio di diversi copisti, e in cui era riuscito a raccogliere una quantità di appunti classici, sele-

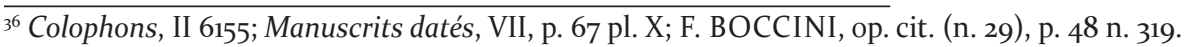

${ }^{37}$ Manuscrits datés, VII, p. 63 pl. CXCVIII e CXCVI.

${ }^{38}$ Colophons, II 5644; Manuscrits datés, VII, p. 65 pl. X-XI.

39 Colophons, V 18555; Manuscrits datés, VII, p. 69 pl. XXIII; F. BOCCINI, op. cit., n. 29, p. 49 n. 324.

${ }^{40}$ Segue l'aggiunta quattrocentesca con la nota di possesso del monastero: Manuscrits datés, VII, p .75 pl. XXIII. Osberno, per stile grafico, ma senza sottoscrizioni, è riconoscibile anche nei codici patristici Avranches, Bibliothèque Municipale, 35 e 103 (in parte): Manuscrits datés, VII, p. 55 pl. XXIV.

${ }_{41}^{4}$ Manuscrits datés, VII, p. 59 pl. CXCV.

${ }^{42}$ Il codice, introdotto da una miniatura architettonica a tutta pagina su sfondo dorato, e completato da eleganti capilettera a intrecci e decorazioni zoomorfe, accoglie i documenti dell'ente, preceduti sui ff. 5r-1or dall'apparizione (revelatio) di s. Michele a s. Auperto e dalla storia dell'insediamento monastico; sui ff. 112V-115r sono stati aggiunti in forma autografa dall'abate de Torigni gli avvenimenti abbaziali del biennio 1155-1157: K. S. B. KEATS-ROHAN, Bibl. Mun. d'Avranches, 210: Cartulary of Mont-Saint-Michel, in Anglo-norman Studies. Proceedings of the Battle conference, 21, 1998 (1999), p. 95-112; U. NILGEN, Le cartulaire du Mont-Saint-Michel et la miniature anglaise, in P. Bouet - M. Dosdat (dir.), Manuscrits et enluminures dans le monde normand ( $X^{e}-X V^{e}$ siècles). Actes du colloque de Cerisy-la-Salle, octobre 1995, Caen, 1999, p. 29-49; E. POULLE - P. BOUET - O. DESBORDES, Cartulaire du Mont-SaintMichel. Fac-similé du manuscrit 210 de la Bibliothèque Municipale d'Avranches, Avranches, 2005; K. S. B. KEATS-ROHAN, The Cartulary of the Abbey of Mont-Saint-Michel, Donington, 2006.

${ }^{43}$ M. PASSALACQUA, Terminologia filologica negli epistolari carolingi: intellettuali e testi, in P. Chiesa (a cura di), Paolino d'Aquileia e il contributo italiano all'Europa carolingia. Atti del Convegno internazionale di studi, Cividale del Friuli-Premariacco, 10-13 ottobre 2002, Udine, 2003 (Libri e Biblioteche, 12), p. 405-420. Per una visione d'insieme della cultura carolingia: R. MCKITTERICK, The Carolingians and the written word, Cambridge, 1989; EAD., History and memory in the carolingian world, Cambridge, 2004.

${ }^{44}$ M. FERRARI, 'In Papia conveniant ad Dungalum', in Italia medioevale e umanistica, 15, 1972, p. 1-52; B. BISCHOFF, Manuscripts and Libraries, op. cit. (n. 18), p. 104 e n. 58 (per la circolazione di Lucrezio), 110-111, 122; S. GAVINELLI, Dungal e l'organizzazione scolastica del regnum Italicum in età carolingia, in D. Mantovani (a cura di), Almum Studium Papiense. Storia dell'Università di Pavia, I/1: Dalle origini all'età spagnola, Milano, 2012, p. 115-128. Il prioritario progetto di insegnamento della Sacra Scrittura è evidente anche nei manoscritti poi donati al cenobio di Bobbio che, come ha evidenziato Bernhard Bischoff, contemplano diversi commenti alla Genesi, ai libri del profeta Esdra, di Salomone, dei Profeti, dei Vangeli e alle Epistole paoline: J. VEZIN, Observations sur l'origine des manuscrits légués par Dungal à Bobbio, in G. Silagi (hrsg.), Paläographie 1981. Colloquium des Comité international de paléographie, München, 1982 (Münchener Beiträge zur mediävistk und Renaissance-Forschung, 32), p. 125-144. 


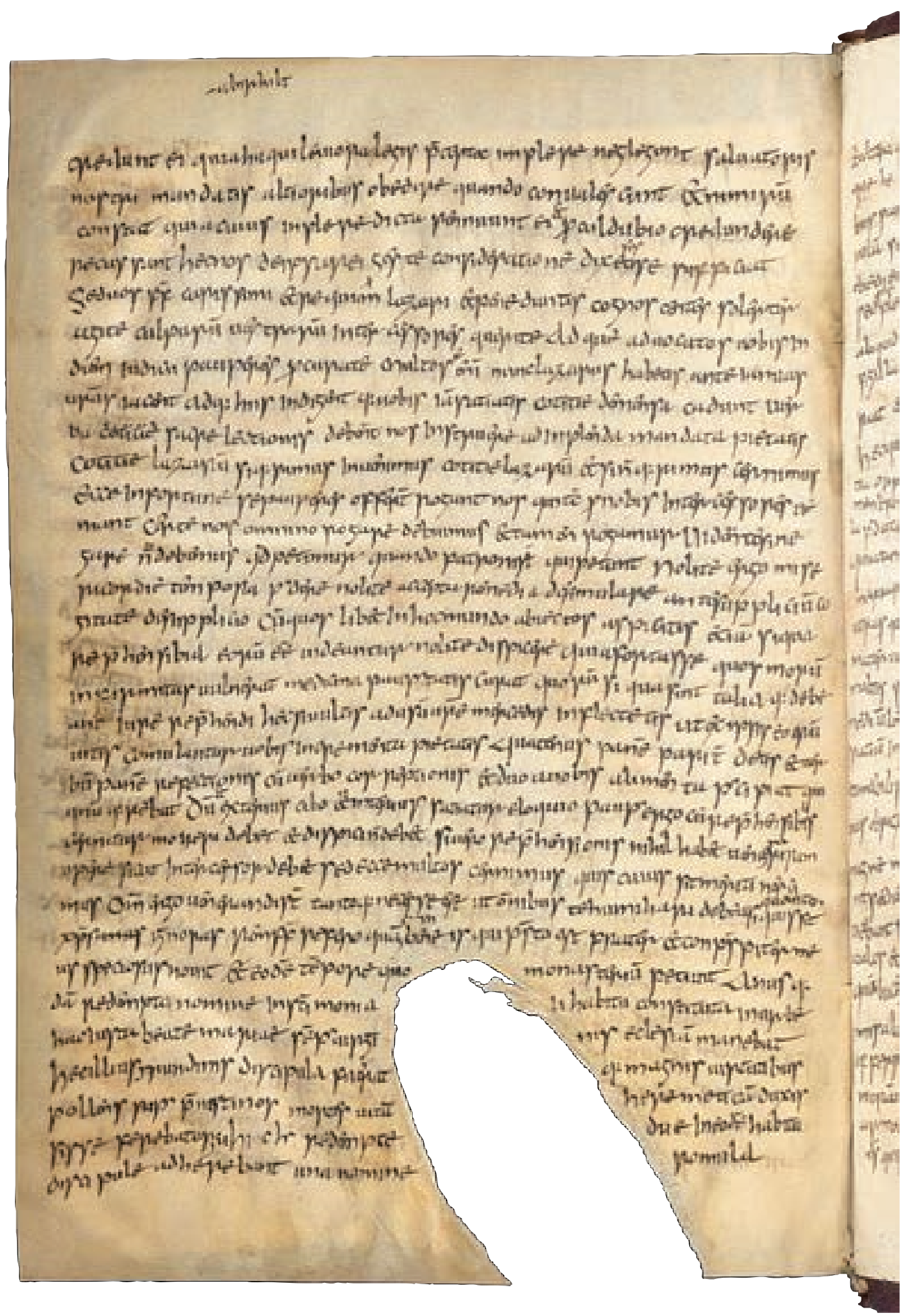

Tav. II. Gregorio Magno, Homeliae XL in evangelia Würzburg, Universitätsbibliothek, M.p.th.f. 45, f. 71v, sec. VIII seconda metà.

zionati su autori rari attinti alla biblioteca della corte (come le Epistolae di Seneca o gli estratti del Calendario del 354), o a quella del monastero di Fulda, e dove non mancano testi di carattere dietetico, medico-terapeutico o agronomico a dimostrazione di come la scienza antica fosse ricercata per affiancare il benessere fisico come presupposto dell'ascesi claustrale ${ }^{45}$. 45 Vi compaiono infatti stralci desunti dagli Scriptores rei rusticae come Palladio e il rarissimo Columella, quest'ultimo in particolare per l'allestimento dei
giardini: G. SCHERRER, Verzeichniss der Handschriften der Stiftsbibliothek von St. Gallen, Halle, 1875, S. 307-309; A. BECCARIA, I codici di medicina
del periodo presalernitano, Roma, 1956 (Storia e letteratura, 53), p. 391-393; H. HOFFMANN, Autographa des früheren Mittelalter, in Deutsches Archiv
für Erforschung des Mittelalters, 57, 2001, S. 1-62: 32-35, in cui si precisa che gli estratti di Columella hanno relazioni testuali con il Milano, Biblioteca
Ambrosiana, L 85 sup., mentre alcuni excerpta di Palladio sono attestati anche in un frammento della fine del sec. IX conservato a Erfurt, Stadtarchiv,
2-III/3, ma copiato a Mainz proprio per denunciare gli stretti legami culturali che intercorsero in quell'arco di tempo tra Mainz e Fulda; S. GAVINELLI,
Tecniche e prodotti della trattatistica agronomica, in G. Archetti (a cura di), La civiltà del pane. Storia, tecniche e simboli dal Mediterraneo all'atlantico.
Atti del convegno internazionale di studio (Brescia, 1-6 dicembre 2014), Spoleto, 2015 (Centro studi longobardi. Ricerche, 1), p. 959-978: 966. Si rilevano
altri interventi correttivi e di postillazione autografa di Walafrido Strabone nell'Orazio Città del Vaticano, Biblioteca Apostolica Vaticana, Reg. lat. 1703, 
Come si è anticipato il numero delle sottoscrizioni con la chiara qualifica di monachus è tuttavia davvero esiguo, ma una delle più antiche attestazioni proviene dall'Italia meridionale. In chiusura dell'elegante Codex Beneventanus dei Vangeli London, British Library, Additional 5463, f. 239v, vergato in onciale della metà del secolo VIII e preceduto dalle tavole miniate delle Concordanze eusebiane (ff. Ir4v), la firma dell'esecutore Lupus monachus, membro della comunità di S. Vincenzo al Volturno, è esibita in vivaci linee di scrittura rosse e nere ${ }^{46}$.

Nel secolo VIII-IX, ai primordi della riforma carolingia, nel monastero di Reichenau si sottoscrive di nuovo come monaco dichiarato Alboinus che, in una fluida minuscola alamannica, fu estensore del commento In Apocalypsin di Primasius di Adrumeto ( $†$ ca. 56o) tradito dal manoscritto Kalrlsruhe, Badische Landesbibliothek, Aug. CCXXII ${ }^{47}$. Ancora più sconfortante è il versante femminile in quanto l'attestazione percentuale rilevabile entro il secolo XII si aggira intorno all' $1 \%$ dei colophons censiti, rendendo calzante l'espressione di genere «Presenze dimenticate» che connota una rassegna di pregevoli contributi di Luisa Miglio e di Marco Palma sulle donne-copiste, le cui fisionomie rimangono spesso sfumate ${ }^{4}$.

Alla seconda metà del secolo VIII risale Burginda - presumibilmente una religiosa di stanza in un monastero dell'In- ghilterra meridionale -, il cui nome, senza alcun stacco grafico, è posto in relazione a una sua lettera di richiesta di suffragi aggiunta in calce al Commento al Cantico dei Cantici di Apponio del Boulogne sur Mer, Bibliothèque Municipale, 74 (82), f. $62 \mathrm{r}^{49}$.

Nella declinazione grafica al femminile si colloca il caso di Guntza, menzionata un paio di volte nel Liber scintillarum di Defensor di Ligugé Würzburg, Universitätsbibliothek, M.p.th.f. 13, ff. 8r, 57v che fu realizzato nel monastero germanico di Kitzingen insieme a due altri scribi dalla scarsa fluidità scrittoria e sotto un forte influsso anglosassonico; il suo nome, trasmesso dalla lettera conclusiva, non presenta alcun rapporto con la responsabilità grafica, come la mancata identificazione degli altri copisti non permette di ipotizzare l'esistenza parallela di una comunità maschile ${ }^{50}$. Al medesimo contesto monastico si riferisce anche Abirhilt, nominata da sola, e nella stessa grafia del testo, nel margine superiore dell'ultima sezione delle Homeliae XL in evangelia di Gregorio Magno Würzburg, Universitätsbibliothek, M.p.th.f. 45, f. 71v, vergate in minuscola anglosassonica da più copisti della seconda metà del secolo VIII [Tav. II $]^{51}$ : essa viene infatti identificata con la badessa del citato monastero di Kitzingen, collocato nell'orbita bonifaciana di Fulda, che fu anche committente di un cospicuo gruppo di codici (Abirhiltgroup).

il più antico testimone dell'opera oraziana copiato da più scribi agli inizi del sec. IX presso l'abbazia alsaziana di Weissenburg: B. BISCHOFF, Eine Sammelhandschrift Walafrid Strabos, in Mittelalterliche Studien, II, Stuttgart, 1967, S. 43, 48, 50; ID., Manuscripts and Libraries, op. cit. (n. 18), p. 122; Virgilio e il Chiostro. Manoscritti di autori classici e civiltà monastica. Abbazia di Montecassino, 8 luglio-8 dicembre 1996, Roma, 1996, p. 113-115 n. 5 (scheda di A. Spotti).

${ }^{46}$ CLA II 162; J. J. JOHN, p. 118. Sul codice: B. FISCHER, Lateinische Bibelhandschriften, op. cit. (n. 12), S. 201; D. H. WRIGHT, The canon tables of the codex Beneventanus and related decoration, in Dunbarton Oaks Papers, 33, 1979, p. 137-155; E. A. LOEW, The Beneventan Script, 2nd ed. by V. Brown, I-II, Roma, 1980 (Sussidi eruditi, 33-34): I, p. 42, 53, 241; II, p. 51; L. DUVAL-ARNOULD, Les manuscrits de San Vincenzo al Volturno, in Miscellanea Cassinense, 51, 1985, p. 354-36o; P. MCGURK, The disposition of numbers in Latin Eusebian canon tables, in Philologia Sacra, 1993, p. 242-258; N. NETZER, Cultural interplay in the eighth century: The Trier Gospels and the makings of a scriptorium at Echternach, Cambridge, 1994 (Cambridge Studies in Palaeography and Codicology, 3), p. 224; E. D’ANGELO, Produzione letteraria e manufatti librari dello scriptorium di San Vincenzo al Volturno, in F. Marrazzi - E. D’Angelo (a cura di), Archeologia della parola. Percorsi e strumenti per a tradizione della memoria di San Vincenzo al Volturno, Cerro al Volturno, 2011, p. 39-42; D. FERRAIUOLO, Tra canone e innovazione. Lavorazione delle epigrafi nella "Langobardia minor" (secoli VIII-X), Firenze, 2013, p. 52. Per lo scriptorium volturnense si veda anche: F. DE RUBEIS, La scrittura a San Vincenzo al Volturno fra manoscritti ed epigrafi, in F. Marazzi (a cura di), San Vincenzo al Volturno. Cultura, istituzioni, economia, Montecassino (Frosinone), 1996 (Miscellanea vulturnense, 3), p. 21-40. Sul f. 76v del prezioso Evangeliario una mano del secolo XIII ha aggiunto una lista dei libri appartenuti alla chiesa di S. Pietro di Benevento intitolata «Ventarius librorum huius ecclesie Sancti Petri», paragonabile all'attività del monaco benedettino Landolfo di S. Sofia di Benevento, che fece acquistare diversi manoscritti per il suo monastero, in genere di carattere biblico, agiografico e liturgico, come indica la nota del secolo XII apposta nella miscellanea del secolo XI Città del Vaticano, Biblioteca Apostolica Vaticana, 4955, f. 209v: E. A. LOEW, The Beneventan Script, op. cit. (Ibidem), p. 53, 78: D. NEBBIAI-DALLA GUARDA, Bibliothèques en Italie jusqu'au XIII siècle. État des sources et premières recherches, in G. Lombardi - D. Nebbiai Dalla Guarda (a cura di), Livres, lecteurs et bibliothèques de l'Italie médiévale (IX'-XVe siècles). Sources, textes et usages. Actes de la Table ronde italo-française (Rome, 7-8 mars 1997) / Libri, lettori e biblioteche dell'Italia medievale (secoli IX-XV). Fonti, testi, utilizzazione del libro. Atti della Tavola rotonda italo-francese (Roma, 7-8 marzo 1997), Paris-Roma, 200o [ma 2001] (Documents, Études et Répertoires, 64), p. 7-129: 33; R. KOTTJE - T. A. ZIEGLER, Verzeichnis der Handschriften mit den Werken des Hrabanus Maurus, Hannover, 2012 (Monumenta Germaniae Historica. Hilfsmittel, 27), S. 211.

${ }^{47}$ CLA VIII 1097; J. J. JOHN, p. 116; B. BISCHOFF, Katalog der festländischen, op. cit. (n. 36), S. 359 n. 1713.

${ }_{48}^{8}$ Colophons, I 2382, II 4442, e 5532-5534, IV 1265 e 12885: A.I. BEACH, Claustration and collaboration between the sexes in the Twelfth-Century scriptorium, in Monks $\mathcal{E}$ nuns, saints $\mathcal{E}$ outcasts: religion in medieval society: essays in honor of Lester K. Little, Ithaca, NY, 200o, p. 57-75: 58. Si veda quindi anche: L. MIGLiO-M. PALMA, Presenze dimenticate (V), in M. D’Agostino - P. Degni (a cura di), Alethes Philia. Studi in onore di Giancarlo Prato, Spoleto, 2010 (Collectanea CISAM, 23), p. 517-523; IID., Presenze dimenticate (VI), in Aevum, 86, 2012, p. 771-782, con bibliografia implicita.

${ }^{49}$ L'epistola, di buona retorica, potrebbe infatti essere stata ripresa da un antigrafo anteriore: CLA VI 738; P. SIMS-WILLIAMS, Religion and Literature in Western England, 60o-800, Cambridge, 1990 (Cambridge Studies in Anglo-Saxon England, 3), p. 211-220; J. J. JOHN, p. 116; L. MIGLIO, "A mulieribus conscriptos arbitror": donne e scrittura, in E. Condello - G. De Gregorio (a cura di), Scribi e colofoni, op. cit. (n. 23), p. 235-266: 238.

${ }^{50}$ CLA IX 1404; A. I. BEACH, Women as Scribes, op. cit. (n. 24), p. 16 dove identifica forse la mano di Guntza in almeno altri tre codici del primitivo appannaggio librario della comunità femminile di Kalburg (Guntza group), i Würzburg, Universitätsbibliothek, M.p.th.f. 17 (Agostino, Commento ai Salmi), M.p.th.f. 28a (Isidoro di Siviglia, Synonyma e materiale agiografico), e M.p.th.f. 78 (Passione degli Apostoli): F. LIFSHITZ, Religious Women in Early Carolingian Francia. A Study of manuscript Transmission and monastic culture, Bronx (NY), 2014, n. 36.

${ }^{51}$ B. BISCHOFF - J. HOFMANN, Libri Sancti Kyliani. Die Würzburger Schreibschule und die Dombibliothek im VIII. und IX. Jahrhundert, Würzburg, 1952 (Quellen und Forschungen zur Geschichte des Bistums und Hochstifts Würzburg, 6), S. 6-14; R. MCKITTERICK, Carolingian, op. cit. (n. 44), p. 169-172; F. LIFSHITZ, Religious Women, op. cit. (n. 5o), dove segnala la presenza di un colophon anonimo nell'Isidoro, De ecclesiasticis officiis Würzburg, Universitätsbibliothek, M.p.th.f. 18 , f. $64 \mathrm{v}$, declinato però al maschile, e quindi forse prodotto presso una cellula parallela attiva all'epoca della prima badessa Hadeloga: B. BISCHOFF - J. HOFMANN, Libri Sancti Kyliani, op. cit. (Ibidem), S. 102-103, 142-148. 
L'esempio più significativo, ampiamente illustrato da Bernhard Bischoff, riguarda però l'abbazia di Chelles, fondata non lontano da Parigi da Batilde, moglie di Clodoveo II, alla metà del secolo VII e dove, alla fine del secolo VIII sotto la guida di Gisla, sorella di Carlo Magno, un gruppo di nove monache («Adruhic, Agnes, Altildis, Angleberta, Eusebia, Girbalda, Gisledrudis, Gislildis, Vera») fu alacremente impegnato nella confezione di tredici manoscritti, per lo più di contenuto patristico, destinati all'arcivescovo Ildebaldo di Colonia ${ }^{52}$. In fondo ai fascicoli dei Köln, Dombibliothek, $63,65,67,97$, secondo un'evidente e precisa suddivisione del lavoro, non resta altro che il mero nome affidato alla memoria della posterità ${ }^{3}$.

La componente aristocratica è riscontrabile pure nella popolazione monastica di S. Salvatore-S. Giulia di Brescia, dove viene ben visualizzata nella sezione memoriale del Liber vitae Brescia, Biblioteca Civica Queriniana, G. I. 7, scritto in carolina verso la metà del secolo IX da una mano principale dell'Italia nord-occidentale ${ }^{54}$. Il fulcro fisico e ideologico del codice, interpretabile come chiara trasposizione scritta dell'identità monastica di un'intera comunità femminile, è marcato da ampie lettere rubricate in maiuscola mista dove, con una pagina-incipit d'impatto (f. $38 \mathrm{r}-\mathrm{v}$ e $42 \mathrm{r}-\mathrm{v}$ del facsimile), inizia una virtuale registrazione di presentiae riferite a puellae di rango dell'aristocrazia funzionariale del regno che, durante l'abbaziato di Amalperga (837-858), venivano affidate al chiostro (Traditio monialium) da illustri padrini, a cominciare da Gisla figlia dell'imperatore Lotario (840-855) e dall'omonima figlia di Ludovico II (844-875), promotore almeno dall' 856 dello sviluppo istituzionale ed economico del cenobio posto in una città eletta a sede privilegiata della sua corte carolingia [Tav. III] 55 .

Tale programmatica icasticità libraria travalicava dunque la sfera spirituale evidenziando gli appoggi vassallatici dell'ente, per quanto non tutte le fanciulle sarebbero state destinate alla permanenza nel microcosmo monastico ${ }^{56}$. Veniva così creato un rapporto scambievole di "reciprocità funzionale" tra l'istituzione e il contesto umano di riferimento per cui l'educazione ricevuta nel monastero assicurava alle fanciulle accolte prestigio, mentre gli affidamenti regali accentuavano l'importanza del cenobio ${ }^{57}$. Sul piano grafico-esecutivo l'assenza di sottoscrizioni da parte dei copisti non consente ovviamente di sbilanciarsi sulla possibile paternità femminile del manoscritto ${ }^{58}$.

Per quanto acefalo e mutilo proviene dallo stesso scriptorium bresciano il Salterio-Collettario Brescia, Biblioteca Civica Queriniana, H. VI. 21, appena punteggiato da poche iniziali a intrecci vegetali che fu completato da un'unica mano carolina verso l'anno Mille, quando il cenobio ancora beneficiava di una circolazione culturale a livello europeo ${ }^{59}$.

${ }_{52}^{2}$ J. J. JOHN, p. 115-120. La scrittura usata a Chelles da Girbada e Gisildis è ad esempio una minuscola del tipo $b$ di Corbie, in alternanza alla scrittura onciale: CLA VI 791 e 792; B. BISCHOFF, Mittelalterliche Studien, I, Stuttgart, 1966, S. 16-34; L. MIGLIO, "A mulieribus conscriptos arbitror", op. cit. (n. 49), p. 236-237; M. BECKWITH PARKES, Their Hands Before Our Eyes. A Closer Look at Scribes, Oxford, 2008 (The Lyell Lectures), p. 8.

${ }_{53}$ Altra figura notevole, ma molto sfumata, è Dulcia che, verso la fine del secolo VIII, si sottoscrisse «Explicit liber premiorum ego Dulcia scripsi et susscripsi istum librum rotarum» nel Laon, Biblothèque Municipale, 423 , f. 79v, copiato in minuscola di tipo a-z forse nel monastero di Notre Dame-la-Profonde a Laon: CLA VI 766; J.J. CONTRENI, The Cathedral School of Laon from 850 to 930. Its Manuscripts and Masters, München, 1978, p. 48-49; J. J. JOHN, p. 110-111, 117; L. MIGLIO, «A mulieribus conscriptos arbitror», op. cit. (n. 49), p. 241 n. 25.

${ }^{54}$ Sul codice si vedano i vari studi anteposti alla riproduzione fotografica: D. Geuenich - U. Ludwig (hrsg.), Der Memorial- und Liturgiecodex von San Salvatore/Santa Giulia in Brescia, Hannover, 2000 (Monumenta Germaniae Historica, Libri memoriales et necrologia. Nova series, 4); U. LUDWIG, Il codice memoriale e liturgico di San Salvatore / Santa Giulia. Brescia e Reichenau, e S. GAVINELLI, La liturgia del cenobio di Santa Giulia in età comunale e signorile attraverso il Liber ordinarius, in G. Andenna (a cura di), Culto e storia in Santa Giulia, Brescia, 2001, p. 103-119 e 121-148: 126-129; EAD., L'omeliario del monastero di San Salvatore-S. Giulia, in Aevum, 78, 2004, p. 345-377:351; F. Crivello - C. Segre Montel (a cura di), Carlo Magno e le Alpi. Viaggio al centro del Medioevo, Milano, 2006, p. 84-85 n. III.2 (scheda di S. Gavinelli).

${ }^{55}$ S. GAVINELLI, La scrittura come simbolo del potere religioso, in G. Andenna (a cura di), Religiosità e civiltà. Le comunicazioni simboliche (secoli IX-XIII). Atti del Convegno Internazionale, Domodossola, Sacro Monte e Castello di Mattarella, 20-23 settembre 2007. «Le Settimane internazionali della Mendola. Nuova Serie 2007-2011», Milano, 2009 (Storia e ricerche), p. 143-180: 155-157. I vari elenchi di monache presenti nel Liber vitae di S. Salvatore-S. Giulia, di non facile ricostruzione prosopografica, hanno consentito ad Hartmut Becher di dimostrare come alla metà del secolo IX il monastero accogliesse circa centoquarantadue religiose, una quantità ragguardevole che ne certificava la solidità economico-finanziaria: H. BECHER, Das königliche Frauenkloster San Salvatore / Santa Giulia im Spiegel seiner Memorialüberlieferung, in Frümittelalterliche Studien, 17, 1983, p. 299-392:303-304; M. DE JONG, Carolingian monasticism: the power of prayer, in R. Mckitterick (ed.), The new Cambridge medieval history, II, Cambridge, 1995, p. 642-643.

${ }^{56}$ Alcune fanciulle transitarono solo dal monastero prima di sposare alcuni membri dell'aristocrazia di appartenenza, come Gisla, figlia di Giuditta e Ludovico il Pio e futura duchessa del Friuli, o l'imperatrice Angelberga, figlia del conte Adalgiso di Parma e quindi fondatrice del monastero di S. Sisto di Piacenza, senza escludere fenomeni più drammatici e diffusi di ratto, come quello occorso alla figlia di Unroch duca del Friuli, fatta rapire dal vescovo Liutwardo di Vercelli (88o-899) per essere data in moglie a un suo nipote: G.P. BOGNETTI, La Brescia carolingia, in Storia di Brescia, I. Dalle origini alla caduta della signoria viscontea (1426), Brescia, 1963, p. 473, 481; C. VIOLANTE, La Chiesa bresciana nel medioevo, in Storia di Brescia, I, p. 999-1124: 1013; G. ARCHETTI, Secundum monasticam disciplinam. San Salvatore di Brescia e le trasformazioni istituzionali di un monastero regio, in Id. (a cura di), Desiderio. Il progetto politico dell'ultimo re longobardo. Atti del Primo convegno internazionale di studio (Brescia, 21-24 marzo 2013), Spoleto, 2015 e 2016 (Centro studi longobardi. Convegni, 1-1.2), p. 631-680: 656. Sul ruolo politico di Angelberga: F. BOUGARD, La cour et le gouvernement de Louis II, 840875, in R. Le Jan (éd.), La royauté et les élites dans l'Europe carolingienne (du début du IXe siècle aux environs de 920), Lille, 1998, p. 249-267; R. CIMINO, Angelberga: il monastero di San Sisto a Piacenza e il corso del fiume Po, in Reti medievali. Rivista, 13/2, 2012, p. 141-162.

57 L. MILIS, Monaci e popolo nell'Europa medievale, Torino, 2003, p. 28, 190.

${ }^{58}$ Oltre alla dispersione della maggioranza del patrimonio manoscritto di S. Salvatore-S. Giulia di Brescia identificato in S. GAVINELLI, L'Omeliario, op. cit. (n. 54), p. 344-36o, pesa l'assenza di elementi relativi alla fisionomia della biblioteca monastica carolingia, presumibilmente riportati nella perduta parte iniziale del Polittico del cenobio Milano, Archivio di Stato, Museo Diplomatico, Cartella V, $7^{1}$ /, l'accertamento (inquisitio) di natura fiscale e patrimoniale redatto in elegante carolina degli inizi del secolo X durante il regno di Berengario I (888-924), dove viene descritto il corredo liturgico di ogni singola cappella per tutte le curtes dipendenti dal cenobio, e da cui si evince la presenza assicurata di almeno qualche libro liturgico, che arrivano però a una ventina per il monastero di Sextunum (presso Rieti): G. PASQUALI, S. Giulia di Brescia, in A. Castagnetti - M. Luzzati - G. Pasquali - A. Vasina (a cura di), Inventari altomedievali di terre, coloni e redditi, Roma, 1979, p. 93-94 in cui la datazione verso la fine del secolo IX suggerita da B. Bischoff a p. 49, viene rettificata da F. BOUGARD, La Justice dans le Royaume d'Italie de la fin du VIII siècle au début du XI siècle, Rome, 1995 (Bibliothèque des Ecoles Françaises d'Athènes et de Rome, 291), p. 395.

${ }^{59}$ K. GAMBER, Codices Liturgici Latini Antiquiores, I-II, Freiburg (Schweiz), 1968² (Spicilegii Friburgensis subsidia, 1/1-2), p. 552 n. 1513; G. BAROFFIO, Iter Liturgicum Italicum, Padova, 1999, p. 37; S. BOYNTON - M. PANTAROTTO, Ricerche sul breviario di Santa Giulia (Brescia, Biblioteca Queriniana, 


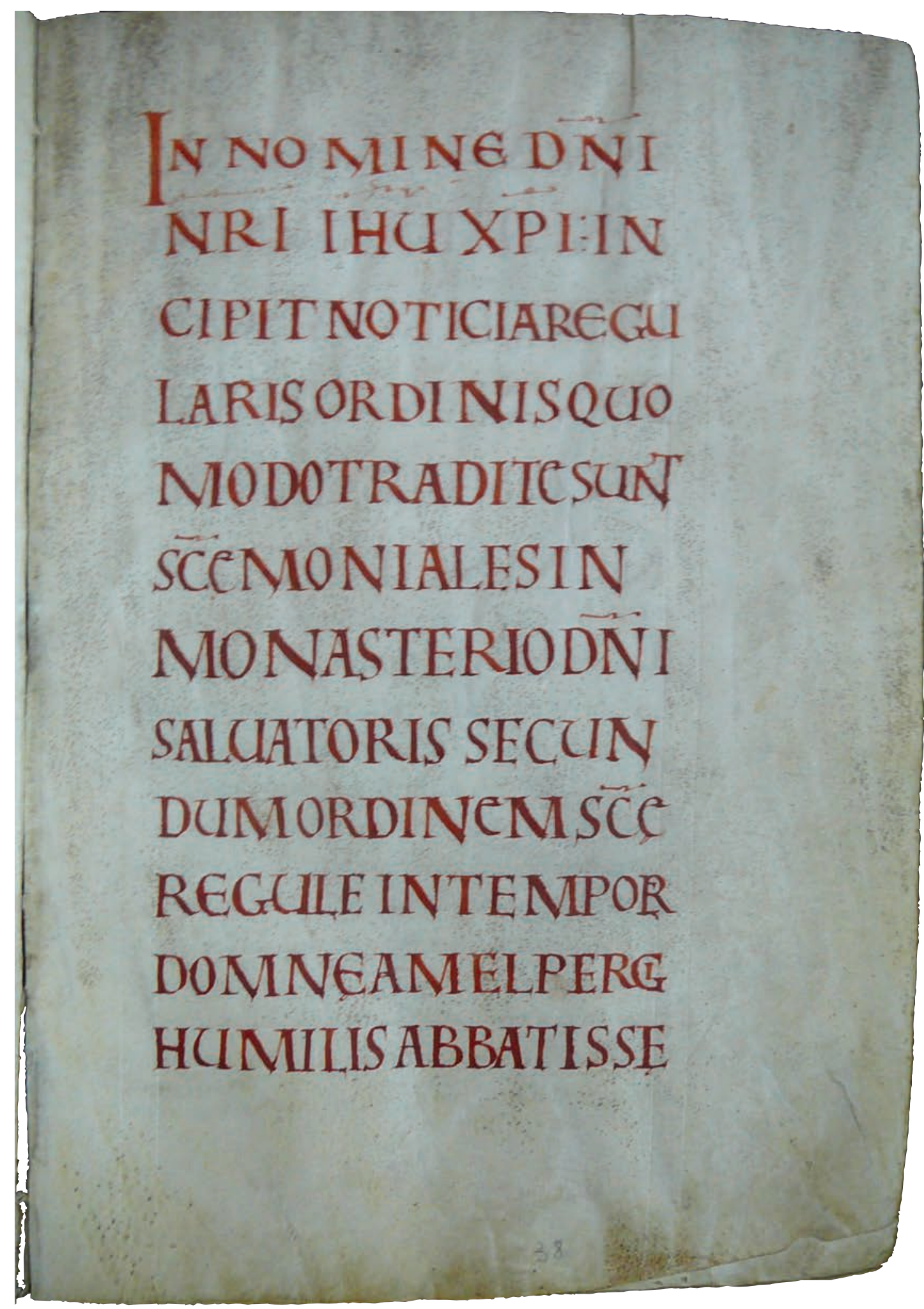

Tav. III. Liber vitae del monastero di S. Salvatore-S. Giulia Brescia, Biblioteca Civica Queriniana, G. I. 7, f. 38r, sec. IX metà.

Nemmeno in questo caso affiorano purtroppo elementi metodologici che autorizzino a ipotizzare una manifattura femminile del documento librario ${ }^{60}$. Al suo interno colpisce però l'aggiunta, appena posteriore (f. 19r), in cui si notifica come la badessa Rolinda (1014-1028) sarebbe stata promo- trice di alcuni importanti interventi architettonici nel cenobio bresciano, nell'intento dunque di rafforzare un chiaro progetto di autorappresentazione monastica rispondente al clima culturale ottoniano ${ }^{6}$. Appare quindi anche coeva la recezione nell'elencazione litanica del Brescia, Biblioteca

ms. H VI 21), in Studi medievali, s. III, 42, 2001, p. 304-318, che datano il codice alla prima metà del sec. XI; S. GAVINELLI, La liturgia, op. cit. (n. 54), p. 129; EAD., L'omeliario, op. cit. (n. 54), p. 351; EAD., Cultura e scrittura, op. cit. (n. 20), p. 34-35.

${ }^{60}$ S. GAVINELLI, Cultura e scrittura, op. cit. (n. 20), p. 34.

${ }^{61}$ L'aggiunta relativa a Rolinda è posta in uno spazio lasciato in precedenza libero dopo alcune prescrizioni sui riti della Settimana Santa, ed esalta i meriti della badessa come fondatrice della chiesa di S. Giovanni evangelista: «In ecclesia qua $(\mathrm{m})$ domna Rolinda abbatissa in honore sancti Iohannis aevangelistae fundavit»: S. GAVINELLI, La liturgia, op. cit. (n. 54), p. 129, cfr. anche G. ARCHETTI, Secundum monasticam disciplinam, op. cit. (n. 56), p. 670-676. 
Civica Queriniana, H. VI. 21, f. 29v delle occorrenze santorali di s. Giulia e di s. Sofia con le tre delle figlie Pistis, Elpis, Agape (le tre virtù teologali, Fede Speranza e Carità) le cui reliquie, come informano fonti più tarde, erano custodite e venerate nella cripta ${ }^{62}$. Oltre a localizzare la fattura del codice denotano un rilancio tutto al femminile dell'ente monastico con un accento orientale e costantinopolitano per s. Sofia, la cui versione latina della vita, ripresa da un originale in greco e responsabile di una migliore veicolazione del culto, sembrerebbe avere avuto una tradizione manoscritta posteriore al secolo IX-X $\mathrm{X}^{6_{3}}$. Nel medesimo Salterio colpiscono le glosse interlineari e marginali, sempre di carattere lessicale e grammaticale, che due mani del secolo XI e XII hanno inserito negli inni sui ff. $23 \mathrm{r}-\mathrm{v}$ e $46 \mathrm{v}$, facendo supporre un congiunto uso scolastico da parte delle monache.

Il mondo delle dominae che gestiscono la cultura investe quindi soprattutto l'aristocrazia germanica che, con il chiostro, mantiene un rapporto costante, dalla formazione giovanile all'approdo protetto nelle fasi più fragili di vedovanza $o$ di senilità ${ }^{64}$. Alla fine dell'Oxford, Bodleian Library, Auct. F. 6. 27, f. 112v si legge infatti un'interessante annotazione che abbraccia tre nomi femminili: «Adelheit Hedwich, Matthilt curiales adulescentulae unum par ... amicitiae» ${ }^{65}$. La menzione di queste fanciulle della casa reale (curiales) non è correlabile con l'attività di copia ma con la lettura condivisa delle fortunate Commedie di Terenzio che, proprio quasi nel- la stessa epoca, erano state riscritte in forma religiosa dalla canonichessa Roswita di Gandersheim $(935-974)^{66}$. Adelaide († 1043) e Matilde († 1025) risultano sorelle del futuro imperatore Ottone III (996-1002), la prima insediata giovanissima come badessa di Quedlinburg e poi di Gandersheim, mentre l'altra, moglie di un conte palatino, aveva fondato il monastero di Braunweiler ${ }^{67}$. La ricostruzione delle biblioteche monastiche di Quedlinburg e di Gandersheim conferma, in effetti, la particolare attenzione riservata da quelle comunità femminili nella promozione degli studi ${ }^{68}$. Edwige, più anziana e loro cugina, è inoltre ricordata più volte nei Casus Sancti Galli per la sua leggendaria cultura da «acutissima Minerva» e per la spregiudicatezza con cui, dopo essere stata promessa a un principe bizantino per questioni dinastiche, aveva fatto le boccacce al pittore incaricato del suo ritratto, facendo così sfumare il matrimonio in favore dell'anziano Burcardo di Svevia ${ }^{69}$. Sua sorella era la badessa Gerberga, maestra di Roswita a Gandersheim, per cui Edwige, dopo essere rimasta vedova, avvertì come naturale la scelta di legarsi ai monaci di S. Gallo insegnando greco, leggendo Virgilio, Orazio e altri classici e assicurando al cenobio libri e suppellettili sacre di pregio, tra cui un paramento ricamato in oro con le nozze di Mercurio con la filologia ${ }^{70}$.

Ai limiti dell'arco cronologico previsto è ascrivibile a mani femminili del secolo XII l'Isidoro, Etymologiae, De natura rerum e altro London, British Library, Harley 3099,

Con tutta probabilità S. Giovanni corrisponde alla cappella, forse rimaneggiata, ricordata anche nell'Ordinario quattrocentesco del monastero Brescia, Biblioteca Civica Queriniana, H. VI. 11 [= Ordinario], f. 29v proprio in connessione con il Sabato Santo, quando i presbiteri di S. Daniele, accompagnati dalla popolazione, dopo essersi portati ad funtes, cioè alla fontana posta nell'atrio per attingere l'acqua destinata alla liturgia battesimale, si recavano alla benedizione del fonte posto in S. Giovanni: «Expletis prophetiis presbiteri et clerici vadant cum ornatu ad funtes. Incipiendo letanias presbiteri et clerici dicant per tres choros, prosequente eos populo usque ad fontem in ecclesia Sancti Iohannis, ubi baptizatur, et facto silentio, intrent ad benedicendum fontis (sic), ut mox (sic) est», cfr. G. ARCHETTI, Per la storia di S. Giulia nel Medioevo. Note storiche in margine al alcune pubblicazioni recenti, in Brixia Sacra. Memorie storiche della diocesi di Brescia, s. III, 5/1-2, 2000, p. 5-44: 26. Per le trasformazioni architettoniche e decorative del monastero bresciano di S. Salvatore - S. Giulia nel secolo X-XI:X. BARRAL I ALTET, Riflessioni sullo stucco come tecnica fondamentale di continuità nell'arte medievale (a proposito del San Salvatore di Brescia), e F. STROPPA, La basilica di San Salvatore: i cicli pittorici altomedievali. Per una rilettura del complesso monastico di Santa Giulia di Brescia, in G. ARCHETTI (a cura di), Desiderio, op. cit. (n. 56), I: p. 681-714 e II.

${ }^{62}$ L'Ordinario, f. 2ov recita infatti: «In archa sancte Iullie sunt tres filie sancte Sophie, secundum quod dicebant domine antique quia viderunt cum oculis suis, scilicet Pistis Elpis Agape et caput matris», cfr. S. GAVINELLI, La liturgia, op. cit. (n. 54), p. 124-125; G. ARCHETTI, Secundum monasticam disciplinam, op. cit. (n. 56), p. 641-646. Secondo la distribuzione degli spazi della chiesa giuliana nella navata centrale, davanti al coro, era collocato l'altare maggiore sotto il quale, nella «confessione», era custodito in tre arche distinte un prezioso bagaglio di reliquie, descritte analiticamente dall'Ordinario, ff. $34 \mathrm{~V}$-35r nel giorno della dedicazione della chiesa maggiore (29/9) come dono dei sovrani fondatori Ansa e Desiderio: G. ARCHETTI, Per la storia, op. cit. (n. 61), p. 23. Sulla possibile traslazione longobarda delle stesse: P. TOMEA, Intorno a S. Giulia. Le traslazioni e le "rapine" dei corpi santi nel regno longobardo (Neustria e Austria), in Culto e storia, op. cit. (n. 54), p. 29-101. Nel 160o, come ricorda la monaca secentesca Angelica Baitelli, storiografa del cenobio, le reliquie furono rimosse dalla cripta per essere collocate nell'altare maggiore della nuova chiesa: A. BAITELLI, Annali Historici dell'edificatione, erettione et dotatione del serenissimo monasterio di S. Salvatore et S. Giulia di Brescia..., in Brescia, MDCLVII, p. 102. Sul personaggio: S. GAVINELLI, Una prospettiva su Desiderio nelle fonti monastiche, in G. Archetti (a cura di), Desiderio, op. cit. (n. 56), p. 553-605: 560-562.

${ }^{6}$ S. GAVINELLI, Santa Sofia e le figlie, Fede, Speranza e Carità dipinte in S. Salvatore - S. Giulia di Brescia?, in G. Archetti (a cura di), Inquirere Veritatem. Studi in memoria di mons. Antonio Masetti Zannini, I, Brescia, 2007 [= Brixia Sacra. Memorie storiche della diocesi di Brescia, s. III, 12/1-2, 2007], p. 83-88.

${ }^{64}$ Famiglia e monastero sono le polarità della condizione femminile medievale, nella misura in cui molte fanciulle potevano essere avviate alla vita claustrale per scongiurare le pesanti doti matrimoniali: J. L. NELSON, Parents, children, and the church in the earlier Middle Ages, in Studies in church history, 31, 1994, p. 81-114; M. DE JONG, In Samuel's image. Child oblation in Early Medieval West, Leiden-New York-Köln, 1996 (Brill's studies in intellectual history, 12), p. 64-66. Questa tendenza venne contrastata in parte dal Capitulare ecclesiastiacum dell'818-819 dove si stabiliva che una fanciulla non potesse prendere il velo prima di avere compiuto venticinque anni: A. DUBREUCQ, Le monachisme féminin dans le nord de la Gaule à l'époque carolingienne, in Les religieuses dans le cloître et dans le monde des origines à nos jours. Actes du Deuxième Colloque International du C.E.R.C.O.R., Poitiers, 29 septembre-2 octobre 1988, Poitiers, 1994, p. 55-71: 60-61; M. DE JONG, In Samuel's image, op. cit. (ibidem), p. 62-64.

${ }_{5}^{65}$ C. VILLA, La «Lectura Terentii», I: Da Ildemaro a Francesco Petrarca, Padova, 1984 (Studi sul Petrarca, 17), p. 99-101.

${ }^{66}$ HROTSWITHAE Opera, ed. H. Homeyer, München-Padernborn-Wien, 1970. Cfr. F. BERTINI, Il «teatro» di Roswita, Genova, 1979; K. BODARWÉ, Hrotswit zwischen Vorbild und Phantom, in M. Boernes - H. Röckelein (hrsg.), Gandersheim und Essen-Vergleichende Untersuchungen zu sächsischen Frauenstiften, Essen, 2006, s. 191-212.

${ }^{67}$ C. VILLA, La «Lectura Terentii», op. cit. (n. 65), p. 103-104. Sul sistema delle fondazioni femminili ottoniane dell'area: G. ALTHOFF, Gandersheim und Quedlinburg. Ottonische Frauenklöster als Herrschafts- und Überlieferungszentren, in Frühmittelalterliche Studien, 25, 1991, S. $123-144$.

${ }^{68}$ K. BODARWÉ, Sanctimoniales litteratae. Schriftlichkeit und Bildung in den ottonischen Frauenkommunitäten Gandersheim, Essen und Quedlinburg, Münster, 2004 (Institut für Kirchengeschichtliche Forschung des Bistums Essen. Quellen und Studien, 10), S. 303-315.

${ }^{69}$ C. VILLA, La «Lectura Terentii», op. cit. (n. 65), p. 104-106, cfr. EKKEHARDI IV Casus Sancti Galli, ed. D. I. von Arx, in MGH, Scriptores, II, Hannoverae, 1829, p. 78-147: 122-123.

$7^{70}$ C. VILLA, La «Lectura Terentii», op. cit. (n. 65), p. 112. 
che risulta copiato in una primitiva littera textualis da otto monache presenti nel monastero di Munsterbilzen (in Belgio vicino a Maastricht) nel primo terzo del secolo, nella cui sottoscrizione sul f. 166r le religiose si definiscono in forma altisonante come «monasterienses dominae» ${ }^{71}$.

L'accento conclusivo di questo colto monachesimo 'al femminile' non può trascurare la rapida menzione della nobile badessa Vekenega di S. Maria di Zara (1095-1111). Appassionata bibliofila come la madre Cika, fondatrice del cenobio nel 1066, risulterebbe infatti la committente dello straordinario Evangeliario Oxford, Bodleian Library, Canonici Bibl. lat. 61 che, in scrittura beneventana del tipo di Bari, e con grandi iniziali dorate a decorazioni geometriche e nastriformi, sarebbe stato eseguito da modelli pugliesi e cassinesi nella contigua comunità maschile di S. Crisogono forse nel 1091 (in occasione della consacrazione della nuova basilica), e presumibilmente anche del Breviario Oxford, Bodleian Library, Canonici lit. 277, richiesto forse per il suo ingresso nel cenobio zaratino come sussidio di preghiera personale adeguato alla sua dignità, $\mathrm{o}$ recepito come eredità della madre $\mathrm{Cika}^{72}$.

\section{APPENDICE: LO SCRIBA-MONACHUS ${ }^{73}$}

Adelbertus monacus, München, Staatsbibliothek, Clm 4503, circa 1065 (Colophons, I 106).

Ademarus monachus Sancti Marcialis, nel Tropario di S. Marziale di Limoges, Paris, Bibliothèque Nationale de France, lat. 1121, ff. 58v, 6or sec. XI primo terzo (Colophons, I 256).

Ademarus indignus monachus, Paris, Bibliothèque Nationale de France, 2400, f. 102V, sec. XI (Colophons, I 257).

Ademarus indignus presbiter et monachus, Berlin, Staatsbibliothek Preussischer Kulturbesitz, lat. Philipp 93, f. 29V (Colophons, I 258).

Adinolfus presbyter et monachus, Città del Vaticano, Biblioteca Apostolica Vaticana, lat. 1274, f. 164r, sec. X (Colophons, I 268).

Aelfwinus monachus et decanus, London, British Library, Cotton Titus, D. XXVII, f. 65v, 1012-1020 (Colophons, I 3760)

Agambaldus indignus vocatus monachus, Città del Vaticano, Biblioteca Apostolica Vaticana, Reg. lat. 281, f. $101 \mathrm{~V}$ (Colophons, I 312).

Albinus monachus Floriacensis, Paris, Bibliothèque Nationale de France, Par. lat. 9576 + Nouv. Acq. lat. 1875, sec. IX metà (Colophons, I 377).
Alboinus monachus inutilis et peccator, Karlsruhe, Badische Landesbibliothek, Aug. CCXXII, f. 176v, ante 822 (Colophons, I 380; J. J. JOHN, p. 116).

Algrimus monachili veste togatus, Bibliothèque Nationale de France, lat. 2136, f. 172v (Colophons, I 452).

Ardericus humilis monachus, Torino, Biblioteca Nazionale Universitaria, D III 16, f. 3o1r sec. XI (Colophons, I 1309).

Arnulfus monachili veste togatus, Wien, Österreichische Nationalbibliothek, 563, sec. XI prima metà (Colophons, I 1431).

Arnulfus fragilis monachus, Madrid, Biblioteca Nacional, Ee 4, 1026 (Colophons, I 1432).

Bernardus monachus, S. Petri Cluniacensis Paris, Bibliothèque Nationale de France, lat. 5071, f. 172v, sec. XI-XII (Colophons, I 2012).

Bertolfus monachus, Aberdeen, University Library, 686, f. 170r, sec. XII (Colophons, I 2195).

Ingelrannus monachus, Paris, Bibliothèque Nationale de France, lat. 11636, ff. 209r, 272v (Colophons, II 3808).

soror Irmengart obtentu Hainrici praepositi, München, Bayerische Staatsbibliothek, Clm 17087 e 17116 (Colophons, II 3929 e 3930).

Gerwigus indignus sacerdos et monachus, London, British Library, 647, sec. XI (Colophons, II 5370).

Gunterius ... et Martinus monachus, Avranches, Bibliothèque Municipale, 98, f. 227v, sec. X-XI (Colophons, II 6155).

monachus Henricus nomine, Boulogne sur Mer, Bibliothèque Municipale, 102, sec. XI (Colophons, II 6319).

Hengilhart monachus, München, Bayerische Staatsbibliothek, Clm 4542, f. 128v (J. J. JOHN, p. 118).

Herimannus sacerdos et monachus, London, British Library, Additional, 22830, 948-994, scritto a Cluny per l'abate Maiolo (Colophons, II 6902).

iuvenis monachus Hugo, Paris, Bibliothèque Nationale de France, lat. 2050, f. 18or, sec. X (Colophons, II 7311).

Liutoldus monachus, Wien, Österreichische Nationalbibliothek, 444 e 1244, circa 1178 (Colophons, IV 1256512566).

Lupus monachus, London, British Library, Additional 5463, f. 239v, sec. VIII metà (J. J. JOHN, p. 118).

Petrus monachus, Grottaferrata, Biblioteca dell'Abbazia, Lit. 73, sec. XI (Colophons, V 15199).

Petrus prepositus ac monachus, Paris, Bibliothèque Mazarine, 1641, ff. 3r, 6r, sec. XI (Colophons, V 15201).

Petrus abbas sub era TCXIV (= 1078), Madrid, Real Academia de la Historia, B. A. H. Cod. 29 (Colophons, V 15204).

\footnotetext{
${ }^{71}$ «Hec sunt nomina illarum quae scripserunt librum istum / Gerdrut, Sibilia, Dierwic, Walderat, Hadewic, / Lugart, o Ota, Cunigunt. Ipse namque scripserunt mo/nasteriensibus dominis quatinus Deum pro eis rogent ut a pe/nis eas liberet et in Paradyso collocet. Quisquis eis ab/stulerit anatematizatus sit. 1134»: A. G. WATSON, Catalogue of Dated, op. cit. (n. 31), p. 132; L. MIGLIO, «A mulieribus conscriptos arbitror», op. cit. (n. 49), p. 239.

${ }^{72}$ L' Oxford, Bodleian Library, Canonici Bibl. lat. 61 viene ricondotto a S. Maria di Zara per la menzione nell'Exultet pasquale (ff. 122r-123r) mentre risulterebbe di livello decorativo inferiore l'altro libro d'ore coevo, il Budapest, Magyar Tudomámyos Académia, K 394, talora identificato come committenza di Vekenega: T.F. KELLY, The Exultet in Southern Italy, Oxford, 1996, p. 257, 273; E. ELBA, La decorazione dei codici in beneventana della Dalmazia tra XI e XIII secolo, in Segno e testo, 4, 2006, p. 107-147; EAD., Lungo le rotte adriatiche: il libro d'ore in beneventana di Budapest e la miniatura pugliese dell'XI secolo, in Rivista di Storia della Miniatura, 12, 2008, p. 45-55; La fortuna dei primitivi. Tesori d'arte, Firenze, 2014, p. 471-472 (scheda di E. Elba); T. GALOVIČ, Scriptura Beneventana-Example of European Calligraphic Script in the Middle Ages. A contribution to the research of manuscrips written in Beneventan script from Croatia, in I. Bratičević - T. Radić (ed.), Classical heritage from the epigraphic to the digital. Academia Ragusina 2009 E 2011, Zagreb, 2014, p. 103-136: 113, 116-125, che riferisce a Cika la confezione dell'Oxford, Bodleian Library, Canonici lit. 277.

${ }_{73}$ Non ho trovato conferme di relazioni scrittorie per Rogerius monachus riportato in Colophons, V16783 come scriba del Salterio Hildesheim, Dombibliothek, Hs. St. God. 1, copiato e miniato tra il 1120 e il 1145 presso l'abbazia inglese di St. Alban in Hertfordshire, restaurato di recente presso il P. Getty Museum di Los Angeles: B. GALLISTL, Der St. Albans Psalter und seine liturgische Verwendung , in Concilium medii aevi, 15, 2012, s. 213-548; ID., Codex and Room. The St. Albans Psalter, in European Research Centre for Book and Paper Conservation Restoration. Newsletter, 2, 2015 (Nov. 2015), p. 4-17; J. GEDDES, Der Albani-Psalter. Eine englische Prachthandschrift des 12. Jahrhunderts für Christina von Markyate, Regensburg, 2005; K. COLLINS - P. KIDD - N. TURNER, The St. Albans Psalter. Painting and Prayer in Medieval England, Los Angeles, 2013.
} 
Petrus monachus, München, Bayerische Staatsbibliothek, Clm 22248, 1141-1191 (Colophons, V 15210).

Rodulphus monachus, Arras, Bibliothèque Municipale, 530, f. ir, sec. XI (Colophons, V 16260).

Ubaldus presbiter et monachus, London, British Library, Additional 15216, sec. XII (Colophons, V 18125).
Werinherus/Wirinherus diaconus et monachus, München, Bayerische Staatsbibliothek, Clm 18523b, f. 158r, 18527, f. 185v, 18646, f. 179r, 19164, f. 141r, sec. XII (Colophons, $\mathrm{V}$ 18747, 18749). 